Cite this: Phys. Chem. Chem. Phys., 2013,

\title{
Influence of adsorption thermodynamics on guest diffusivities in nanoporous crystalline materials $\dagger$
}

15, 7994

Received 31st January 2013, Accepted 26th March 2013

DOI: $10.1039 / \mathrm{c3cp50449b}$

www.rsc.org/pccp

\author{
Rajamani Krishna* and Jasper M. van Baten
}

\begin{abstract}
Published experimental data, underpinned by molecular simulations, are used to highlight the strong influence of adsorption thermodynamics on diffusivities of guest molecules inside ordered nanoporous crystalline materials such as zeolites, metal-organic frameworks (MOFs), and zeolitic imidazolate frameworks (ZIFs). For cage-type structures (e.g. LTA, CHA, DDR, and ZIF-8), the variation of the free energy barrier for inter-cage hopping across the narrow windows, $-\delta F_{i}$, provides a rationalization of the observed strong influence of pore concentrations, $c_{i}$, on diffusivities. In open structures with large pore volumes (e.g. FAU, IRMOF-1, CuBTC) and within channels (MFI, BEA, MgMOF-74, MIL-47, MIL-53), the pore concentration $\left(c_{i}\right)$ dependence of the self- $\left(D_{i, \text { self }}\right)$, Maxwell-Stefan $\left(\theta_{i}\right)$, and Fick $\left(D_{i}\right)$ diffusivities are often strongly dictated by the inverse thermodynamic correction factor, $1 / \Gamma_{i} \equiv \partial \ln c_{i} / \partial \ln p_{i}$ i the magnitudes of the diffusivities are dictated by the binding energies for adsorption. For many guesthost combinations $\theta_{i}-c_{i}$ dependence is directly related to the $1 / \Gamma_{i}$ vs. $c_{i}$ variation. When molecular clustering occurs, we get $1 / \Gamma_{i}>1$, causing unusual $D_{i}$ vs. $c_{i}$ dependencies. The match, or mis-match, between the periodicity of the pore landscape and the conformations of adsorbed chain molecules often leads to non-monotonic variation of diffusivities with chain lengths.
\end{abstract}

\section{Introduction}

Ordered crystalline nanoporous materials such as zeolites (crystalline aluminosilicates), metal-organic frameworks (MOFs), and zeolitic imidazolate frameworks (ZIFs) are of potential use in emerging technologies for separation of a wide variety of mixtures $\mathrm{CO}_{2}-\mathrm{N}_{2}$, $\mathrm{CO}_{2}-\mathrm{CH}_{4}, \mathrm{CO}_{2}-\mathrm{CO}-\mathrm{CH}_{4}-\mathrm{H}_{2}, \mathrm{CO}_{2}-\mathrm{H}_{2}, \mathrm{CH}_{4}-\mathrm{H}_{2}, \mathrm{O}_{2}-\mathrm{N}_{2}$, natural gas, alkane-alkene, alkyne-alkene, and hydrocarbon isomers. ${ }^{1-15}$ The separation performance is influenced by a combination of adsorption and diffusion characteristics, which in turn are dictated by a wide variety of factors that include pore size, pore topology, connectivity, and interactions (both van der Waals and electrostatic) of the guest molecules with the framework atoms. Membrane separations are often reliant on diffusion selectivities in order to achieve the required separation performance that are not achievable solely on the basis of differences in adsorption strengths. ${ }^{16,17}$

Van't Hoff Institute for Molecular Sciences, University of Amsterdam, Science Park 904, 1098 XH Amsterdam, The Netherlands. E-mail: r.krishna@uva.nl;

Fax: +3120 525 5604; Tel: +31206270990

$\dagger$ Electronic supplementary information (ESI) available: Pore landscapes, structural details of a variety of mesoporous and microporous structures (zeolites, MOFs, ZIFs), molecular simulation data for the loading dependence of self- and Maxwell-Stefan diffusivities, along with the thermodynamic correction factors and isosteric heats of adsorption, and video animation demonstrating the rotation of benzene at the intersections of MFI. See DOI: 10.1039/c3cp50449b
While the measurements, simulations, and estimations of adsorption thermodynamics are relatively well established, the proper estimation of diffusivities of guest molecules continues to provide major challenges to fundamental researchers and practitioners alike. ${ }^{1,18-24}$ The major objective of this article is to highlight the interplay between adsorption thermodynamics and diffusivities of guest molecules. By performing a detailed analysis of available experimental data, supplemented by molecular simulations, we aim to highlight the factors that strongly influence the guest diffusivity in nanoporous materials, such as binding energies, thermodynamic correction factors, commensurateness of molecular dimensions with periodicity of the framework, molecular clustering, and free-energy barriers exert on both the magnitude and concentration dependence of diffusivities. The precise nature of the influence of each of these factors depends on the pore dimensions and pore topology. The characteristic pore dimensions are either in the microporous $\left(d_{\mathrm{p}}<20 \AA\right)$ or mesoporous ranges $\left(20 \AA<d_{\mathrm{p}}\right.$ $<500 \AA$ ). Several types of channel topologies are encountered in practice, including one-dimensional (1D) channels (e.g. AFI, LTL, MIL-47, MIL-53(Cr), MgMOF-74, and BTP-COF), 1D channels with side pockets (e.g. MOR), intersecting channels (e.g. MFI, BEA, FER, ISV BOG, Zn(bdc)dabco), cavities with large windows (e.g. FAU, IRMOF-1, CuBTC), and cages separated by narrow windows (e.g. LTA, CHA, DDR, TSC, ERI, ITQ-29, ZIF-8). 
Table 1 Salient structural information for zeolites, MOFs and COFs

\begin{tabular}{|c|c|c|c|c|}
\hline Structure & Topology & $\begin{array}{l}\text { Fractional pore } \\
\text { volume, } \phi\end{array}$ & $\begin{array}{l}\text { Pore volume } \\
\left(\mathrm{cm}^{3} \mathrm{~g}^{-1}\right)\end{array}$ & $\begin{array}{l}\text { Framework } \\
\text { density }\left(\mathrm{kg} \mathrm{m}^{3}\right)\end{array}$ \\
\hline AFI & 12-ring 1D channels of $7.3 \AA ̊$ size & 0.274 & 0.159 & 1730 \\
\hline BEA & Intersecting channels of two sizes: 12 -ring of $6.1 \AA \times 6.8 \AA$ and 10-ring of $5.6 \AA \times 6.7 \AA$ & 0.408 & 0.271 & 1509 \\
\hline BOG & Intersecting channels: 12 -ring $6.8 \AA \times 7.4 \AA$ and 10 -ring of $5.6 \AA \times 5.8 \AA$ & 0.374 & 0.241 & 1996 \\
\hline CHA & $316 \AA^{3}$ cages separated by $3.77 \AA \times 4.23 \AA$ size windows & 0.382 & 0.264 & 1444 \\
\hline DDR & $277.8 \AA^{3}$ cages separated by $3.65 \AA \times 4.37 \AA$ size windows & 0.245 & 0.139 & 1760 \\
\hline $\begin{array}{l}\text { FAU-Si } \\
\text { (all-silica) }\end{array}$ & $790 \AA^{3}$ cages separated by $7.4 \AA$ size windows & 0.439 & 0.328 & 1338 \\
\hline FER & $\begin{array}{l}\text { 10-ring channels of } 4.6 \AA \times 5.4 \AA \text { size intersect with 8-ring channels of } \\
3.5 \AA \times 4.8 \AA \text { size }\end{array}$ & 0283 & 0.16 & 1772 \\
\hline MOR & $\begin{array}{l}\text { 12-ring channels of } 6.5 \AA \times 7 \AA \text { size are connected to side pockets via 8-ring } \\
\text { channels of } 3.4 \AA \times 4.8 \AA \text { size }\end{array}$ & 0.285 & 0.166 & 1714 \\
\hline $\mathrm{NaY}$ & $790 \AA^{3}$ cages separated by $7.4 \AA$ size windows & 0.41 & 0.303 & 1347 \\
\hline $\mathrm{NaX}$ & $790 \AA^{3}$ cages separated by $7.4 \AA$ size windows & 0.40 & 0.280 & 1421 \\
\hline ISV & Intersecting channels of two sizes: 12 -ring of $6.1-6.5 \AA$ and 12-ring of 5.9-6.6 & 0.426 & 0.278 & 1533 \\
\hline ITQ-29 & $678 \AA^{3}$ cages separated by $4 \AA \times 4.22 \AA$ size windows & 0.405 & 0.283 & 1433 \\
\hline $\begin{array}{l}\text { LTA-Si } \\
\text { (all-silica) }\end{array}$ & $743 \AA^{3}$ cages separated by $4.11 \AA \times 4.47 \AA$ size windows & 0.399 & 0.310 & 1285 \\
\hline LTA- 4 A & $694 \AA^{3}$ cages separated by $4 \AA \times 4.6 \AA$ size windows & 0.375 & 0.245 & 1529 \\
\hline LTA-5A & $702 \AA^{3}$ cages separated by $4 \AA \times 4.6 \AA$ size windows & 0.38 & 0.252 & 1508 \\
\hline LTL & 12-ring $1 \mathrm{D}$ channels & 0.277 & 0.17 & 1627 \\
\hline MFI & 10-ring intersecting channels of 5.4-5.5 $\AA$ and 5.4-5.6 $\AA$ size & 0.297 & 0.165 & 1796 \\
\hline MTW & 12-ring 1D channels of $5.6 \AA \times 6.8 \AA$ size & 0.215 & 0.111 & 1935 \\
\hline BTP-COF & 1D hexagonal-shaped channels of $34 \AA$ & 0.752 & 1.79 & 420 \\
\hline CuBTC & $\begin{array}{l}\text { Large cages are inter-connected by } 9 \AA \text { windows of square cross-section. } \\
\text { The large cages are also connected to tetrahedral-shaped pockets } \\
\text { of } c a .6 \AA \text { size through triangular-shaped windows of } 4.6 \AA \text { size }\end{array}$ & 0.759 & 0.863 & 879 \\
\hline IRMOF1 & $\begin{array}{l}\text { Two alternating, inter-connected, cavities of } 10.9 \AA \text { and } 14.3 \AA \\
\text { with window size of } 8 \AA \text {. }\end{array}$ & 0.812 & 1.369 & 593 \\
\hline MOF-177 & $\begin{array}{l}\text { Six diamond-shaped channels (upper) with diameter of } 10.8 \AA \text { surround a pore } \\
\text { containing eclipsed } \mathrm{BTB}^{3-} \text { moieties. }\end{array}$ & 0.840 & 1.968 & 427 \\
\hline MgMOF-74 & 1D hexagonal-shaped channels of $11 \AA$ & 0.708 & 0.782 & 905 \\
\hline ZnMOF-74 & 1D hexagonal-shaped channels of $11 \AA$ & 0.709 & 0.582 & 1219 \\
\hline MIL-47 & 1D diamond-shaped channels of $8.5 \AA$ & 0.608 & 0.606 & 1004 \\
\hline $\begin{array}{l}\text { MIL-53 } \\
\text { (Cr)-lp }\end{array}$ & 1D lozenge-shaped channels of $8.5 \AA$ & 0.539 & 0.518 & 1041 \\
\hline $\mathrm{Zn}(\mathrm{bdc}) \mathrm{dabco}$ & $\begin{array}{l}\text { There exist two types of intersecting channels: } 7.5 \AA \times 7.5 \AA \text { along the } x \text {-axis and } \\
\text { channels of } 3.8 \AA \times 4.7 \AA \text { along } y \text { and } z \text { axes. }\end{array}$ & 0.662 & 0.8 & 826 \\
\hline ZIF-8 & $1168 \AA^{3}$ cages separated by $3.26 \AA$ size windows & 0.476 & 0.515 & 924 \\
\hline
\end{tabular}

The ESI $\dagger$ accompanying this publication provides all structural details of all materials considered in this perspective; some salient data are summarized in Table 1.

\section{Adsorption isotherms, saturation capacities, molecule-wall, and molecule- molecule interactions}

In practice, separation processes are often conducted under processing conditions such that the concentrations and loadings inside the pores are large enough to influence the diffusion characteristics. Precisely how diffusivities of guest molecules are altered with increasing loadings is often strongly influenced by the adsorption isotherms. To elucidate this influence, we begin by considering the pure component adsorption isotherms for $\mathrm{CO}_{2}, \mathrm{CH}_{4}, \mathrm{C}_{2} \mathrm{H}_{6}, \mathrm{C}_{3} \mathrm{H}_{8}, n \mathrm{C}_{4} \mathrm{H}_{10}$, and $n \mathrm{C}_{6} \mathrm{H}_{14}$ in some commonly used zeolites and MOFs. For consistent comparisons, we use Configurational-Bias Monte Carlo (CBMC) simulations of the isotherms. The most commonly used measures of the adsorbate phase loadings are (1) molar loadings, $q_{i}$, expressed as moles adsorbate per kg of the framework, and (2) $\Theta_{i}$, molecules adsorbed per unit cell or per cage. However, for comparison purposes it is much more convenient and useful to use adsorbed phase pore concentrations, $c_{i}$, defined in terms of the accessible pore volume $V_{\mathrm{p}}$ (see values in Table 1)

$$
c_{i}=q_{i} / V_{\mathrm{p}}
$$

For all six guest molecules, the CBMC data in Fig. 1 show that the saturation capacities, $c_{i \text {,sat }}$, are nearly the same for all structures. The value of $c_{i \text {,sat }} \approx 32,28,20,15,10$, and $7 \mathrm{kmol} \mathrm{m}^{-3}$, respectively, for $\mathrm{CO}_{2}, \mathrm{CH}_{4}, \mathrm{C}_{2} \mathrm{H}_{6}, \mathrm{C}_{3} \mathrm{H}_{8}, n \mathrm{C}_{4} \mathrm{H}_{10}$, and $n \mathrm{C}_{6} \mathrm{H}_{14}$. These $c_{i \text {,sat }}$ values agree closely with the values of the molar densities of the saturated liquid at $300 \mathrm{~K}$ at the pressure at which pore saturation is attained. Barrer and Sutherland ${ }^{25}$ were the first to suggest that the molar density of the liquid phase is a good estimate of $c_{i \text {,sat }}$ of a variety of guest species in the FAU zeolite; this suggestion was latter used by Golden and Sircar for adsorption in MFI. ${ }^{26}$ This estimation is particularly useful for light gases that have poor adsorption strength; in such cases it is difficult, if not impossible, to attain saturation conditions in adsorption isotherm measurements even when operating at high pressures. ${ }^{27,28}$ 
(a)

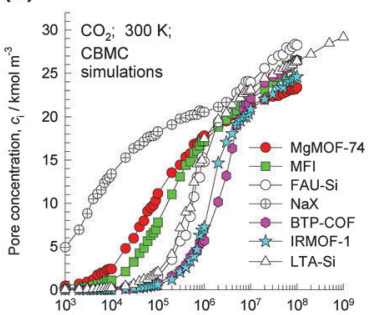

(c)

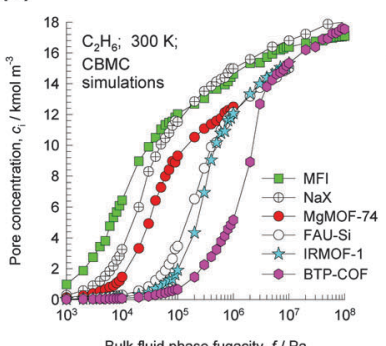

Bulk fluid phase fugacity, $f_{i} / \mathrm{Pa}$

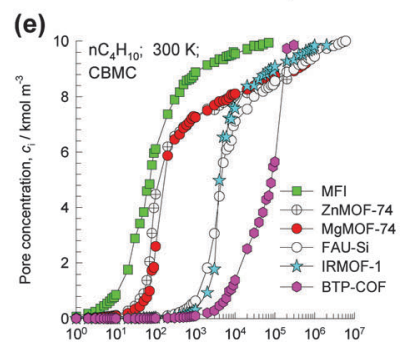

Bulk fluid phase fugacity, $\mathrm{f}_{\mathrm{i}} / \mathrm{Pa}$ (b)

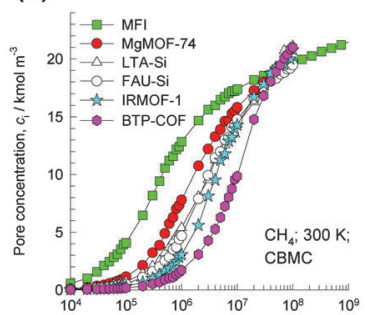

(d)

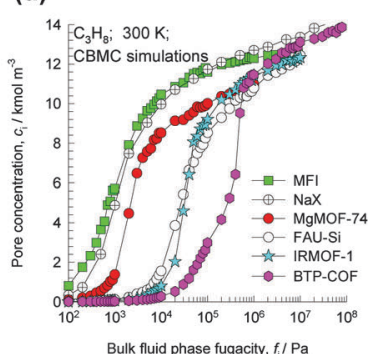

(f)

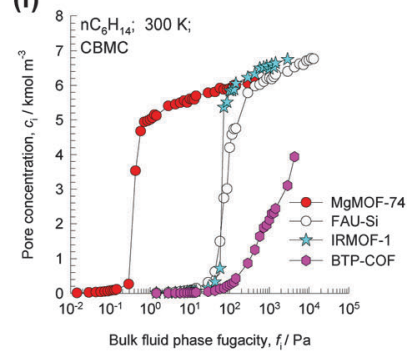

Fig. 1 CBMC simulations of adsorption isotherms for (a) $\mathrm{CO}_{2}$, (b) $\mathrm{CH}_{4}$, (c) $\mathrm{C}_{2} \mathrm{H}_{6}$, (d) $\mathrm{C}_{3} \mathrm{H}_{8}$, (e) $n \mathrm{C}_{4} \mathrm{H}_{10}$, and (f) $n \mathrm{C}_{6} \mathrm{H}_{14}$ in zeolites and MOFs at $300 \mathrm{~K}$.

As the saturation capacity $c_{i \text {,sat }}$ is approached the diffusivities for any guest molecule tend to decrease significantly in magnitude. This is best illustrated by the Molecular Dynamics (MD) simulation data of the self-diffusivities, $D_{i \text {,self }}$, determined by analysing the mean square displacement of individual molecules ${ }^{1}$

$$
D_{i, \text { self }}=\frac{1}{2} \lim _{\Delta t \rightarrow \infty} \frac{1}{n_{i} \Delta t} \sum_{l=1}^{n_{i}}\left(\boldsymbol{r}_{l, i}(t+\Delta t)-\boldsymbol{r}_{l, i}(t)\right)^{2}
$$

The values obtained from eqn (2) apply to each coordinate direction. For structures such as MFI, NaX, IRMOF-1, LTA, and CHA, that have three-dimensional (3D) connectivity, the reported values are those obtained by averaging over each of the three coordinate directions. For one-dimensional channel structures such as MIL-47, MIL-53, MgMOF-74, and BTP-COF the reported values are along the channel axes. The data in Fig. 2 show that for each guest-host combination, the $D_{i \text {,self }}$ suffers a strong reduction, often by about one to two orders of magnitude, as the pore concentrations are increased to values approaching $c_{i \text {,sat }}$. A further point to note from the data in Fig. 2

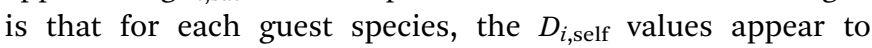
converge to approximately the same values in different structures as the $c_{i, \text { sat }}$ value is approached.

While the self-diffusivities are arguably the easiest to determine using either MD or experimental techniques such as NMR or QENS, ${ }^{1,20}$ the interpretation and modelling of the concentration

(a)

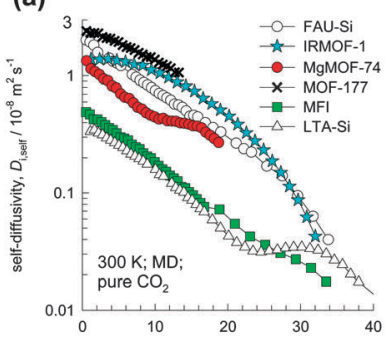

(c)
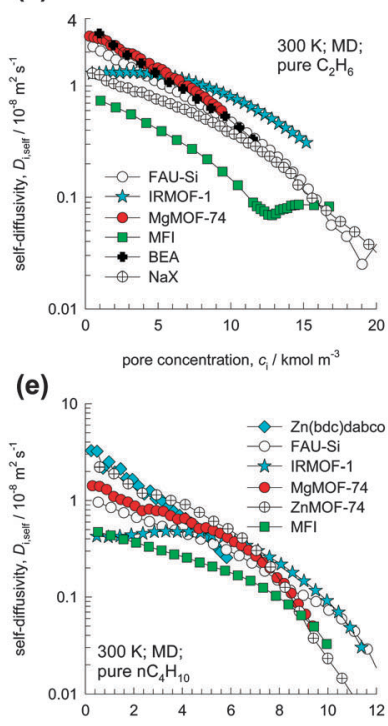

pore concentration, $c_{\mathrm{i}} / \mathrm{kmol} \mathrm{m}^{-3}$

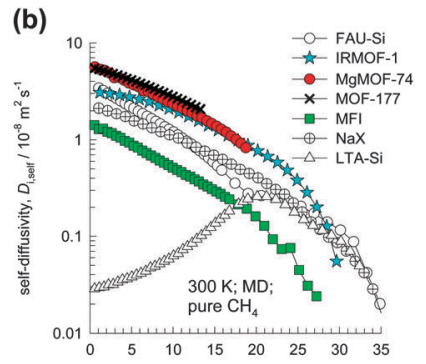

(d)

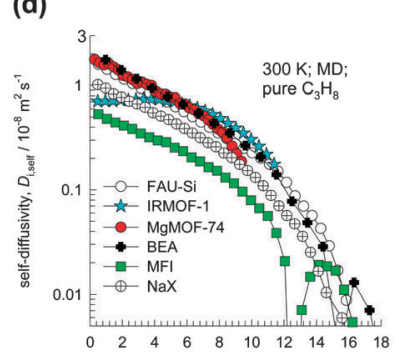

pore concentration, $c_{\mathrm{i}} / \mathrm{kmol} \mathrm{m}^{-3}$

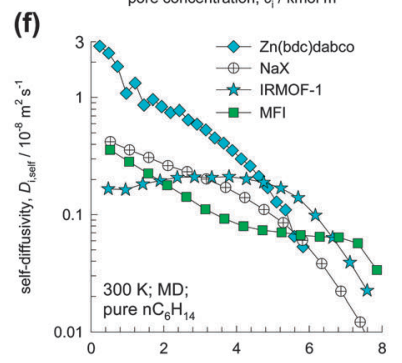

pore concentration, $c_{\mathrm{i}} / \mathrm{kmol} \mathrm{m}^{-3}$

Fig. 2 MD simulations of $D_{i, \text { self }}$ for (a) $\mathrm{CO}_{2}$, (b) $\mathrm{CH}_{4}$, (c) $\mathrm{C}_{2} \mathrm{H}_{6}$, (d) $\mathrm{C}_{3} \mathrm{H}_{8}$, (e) $n \mathrm{C}_{4} \mathrm{H}_{10}$, and (f) $n \mathrm{C}_{6} \mathrm{H}_{14}$ in zeolites and MOFs at $300 \mathrm{~K}$, as a function of the pore concentrations $c_{i}$, determined in terms of the accessible pore volumes $V_{p}$. The diffusivity data for $\mathrm{Zn}(\mathrm{bdc})$ dabco are determined along the $7.5 \AA \times 7.5 \AA$ square channels.

dependencies of $D_{i \text {,self }}$ is not simple, primarily because they reflect a combination of molecule-molecule and molecule-wall interactions. Each of these interactions depends, in a complex manner, on a wide variety of factors. A much clearer understanding of pore diffusion is obtained by de-coupling these two interactions, and examining each separately. The Maxwell-Stefan (M-S) diffusivity, $Ð_{i}$, characterizes molecule-wall interactions; these can be determined from MD simulations by monitoring the mean square displacement of an ensemble of molecules ${ }^{1,20}$

$$
\bigoplus_{i}=\frac{1}{2} \lim _{\Delta t \rightarrow \infty} \frac{1}{n_{i} \Delta t}\left(\sum_{l=1}^{n_{i}}\left(\boldsymbol{r}_{l, i}(t+\Delta t)-\boldsymbol{r}_{l, i}(t)\right)\right)^{2}
$$

If we define the self-exchange coefficient $\bigoplus_{i i}$ as a diffusivity characterising molecule-molecule interactions, we get the formula for addition of the individual resistances ${ }^{20,21,29}$

$$
1 / D_{i, \text { self }}=1 / \bigoplus_{i}+1 / \bigoplus_{i i}
$$

For $\mathrm{CH}_{4}$, as illustration, MD simulations of the values of $D_{i, \text { self }}, \emptyset_{i}$, along with the $\bigoplus_{i i}$ calculated using eqn (4), are shown in Fig. 3 for six different host structures. At any pore concentration, 
(a)

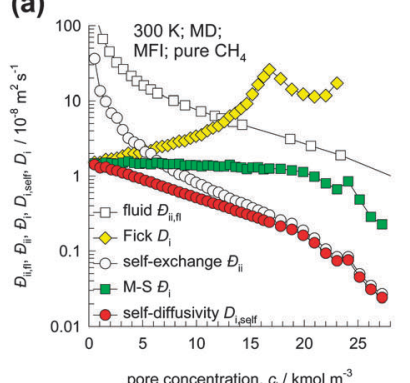

pore concentration, $c_{1} / \mathrm{kmol} \mathrm{m}^{-3}$

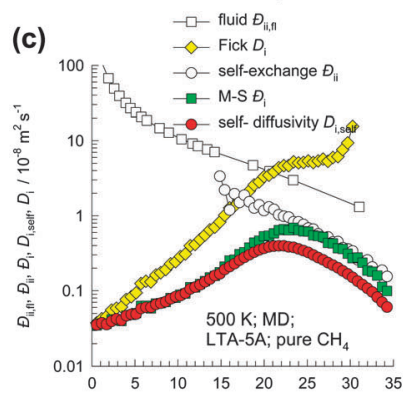

pore concentration, $c_{i} / \mathrm{kmol} \mathrm{m}^{-3}$

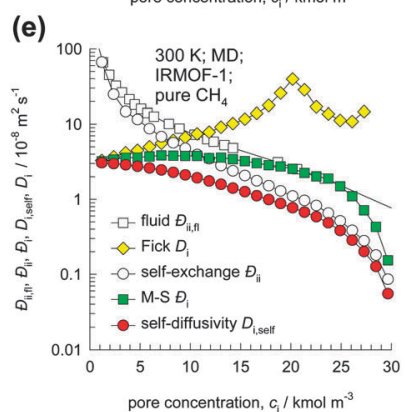

(b)

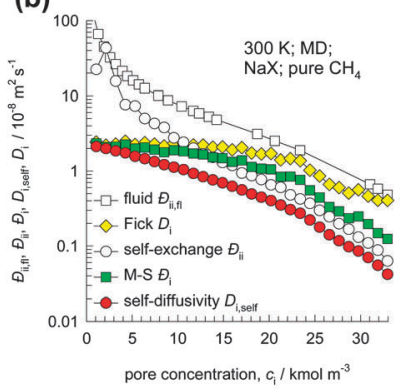

(d)

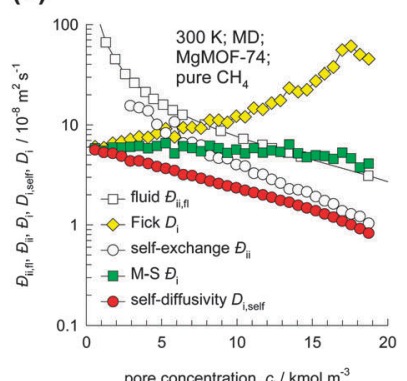

(f)

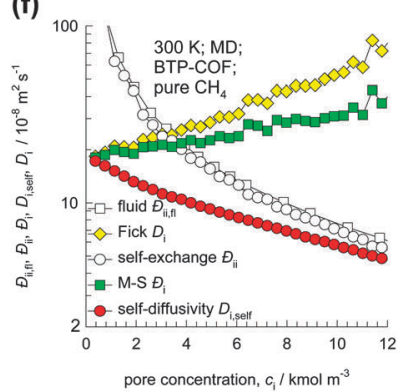

Fig. 3 MD simulations of $D_{i, \text { self, }} \bigoplus_{i}, D_{i i}$ and $D_{i}$, for $\mathrm{CH}_{4}$ in (a) MFI, (b) $\mathrm{NaX}$, (c) LTA5A, (d) MgMOF-74, (e) IRMOF-1, and (f) BTP-COF, as a function of the pore concentrations $c_{i}$. Also indicated by open square symbols are the values of the fluid phase self-diffusivities, $\Theta_{i, \text { fll }}$, determined from MD simulations using the same force field parameters. Note that the data for LTA-5A are obtained at $500 \mathrm{~K}$, whereas the rest of the data are obtained at $300 \mathrm{~K}$.

$D_{i, \text { self }} \leq D_{i}$; this is because individual jumps of molecules are correlated due to re-visitation of sorption sites that have been recently vacated. The extent of correlations is captured by the self-exchange coefficient $D_{i i}$. For all structures, in the limit of low pore concentrations, $c_{i} \rightarrow 0 ; q_{i} \rightarrow 0$, molecule-molecule interactions are of negligible importance, and $\Xi_{i i}$ is significantly higher than $D_{i, \text { self }}$ and $D_{i}$. Correlations become increasingly significant as the pore concentrations are increased, and this is evidenced by the observation that $D_{i i}$ decreases more sharply with increasing $c_{i}$, than the M-S diffusivity $D_{i}$. As pore saturation conditions are approached, the diffusion characteristics are dominated by correlation effects..$^{30,31}$

The relative importance of molecule-wall and moleculemolecule interactions is different for each structure. In mesoporous materials, the self-exchange coefficient $D_{i i}$ can be identified with the self-diffusivity in the fluid phase, $D_{i i, f l}$, over the entire range of pore concentrations. ${ }^{32}$ This is demonstrated by the data for BTP-COF that has 1D hexagonal-shaped channels of $34 \AA$ size; see Fig. 3 f. For BTP-COF we note that $D_{i i} \approx D_{i i, \mathrm{fl}}$

over the entire range of concentrations; this conclusion is valid for all guest molecules in mesoporous channels. ${ }^{29,32-34}$ For microporous materials, the values of $\emptyset_{i i}$ are lower than $\emptyset_{i i, \mathrm{fl}}$ by a factor $F$; this factor is practically constant over the entire range of pore concentrations. ${ }^{17,32}$ The extent of lowering depends on pore size, pore topology and connectivity. In the MFI zeolite, the factor $F \approx 0.1$. For MgMOF-74, NaX, and IRMOF-1, the factor $F$ falls in the range 0.4-0.7.

Correlations are relatively weak for LTA-5A in which the diffusivities are largely dictated by hopping of molecules, oneat-a-time, across $4 \AA \times 4.58 \AA$ size windows ( $c f$. Fig. $4 \mathrm{a}$ ); this is evidenced by the fact that $D_{i, \text { self }} \approx D_{i}$ for $c_{i}<20 \mathrm{kmol} \mathrm{m}^{-3}$; see Fig. 3c. Correlations in LTA-5A become of importance only at higher pore concentrations; these conclusions are also valid for other cage-type structures such as CHA, DDR, ITQ-29, ERI, and ZIF-8. ${ }^{17,35}$

There is no experimental procedure for direct determination of the $D_{i}$. Transient uptake and chromatographic experiments yield the Fick diffusivity $D_{i}$, also termed "transport" diffusivity, that relates the flux $N_{i}$ of species $i$ to the gradient of the molar loadings, $q_{i}$, or pore concentration, $c_{i}$

$$
N_{i}=-\rho D_{i} \nabla q_{i}=-\phi D_{i} \nabla c_{i}
$$

where $\phi$ is the fractional pore volume. The $D_{i}$ are related to the M-S diffusivities by the thermodynamic factor $\Gamma_{i}$

$$
D_{i}=\emptyset_{i} \Gamma_{i} ; \Gamma_{i} \equiv \frac{c_{i}}{f_{i}} \frac{\partial f_{i}}{\partial c_{i}}=\frac{q_{i}}{f_{i}} \frac{\partial f_{i}}{\partial q_{i}}
$$

The M-S diffusivity $\emptyset_{i}$ is often termed the "corrected" diffusivity because formula (6) suggests that adsorption thermodynamic effects have been "factored out". The values of $\Gamma_{i}$ can be determined by analytic differentiation of fits to the adsorption isotherms, such as those depicted in Fig. 1. For a proper understanding of the concentration dependence of the diffusivities, we need the isotherm fits to be good representations over the entire range of concentrations, and not just in the Henry regime $\left(c_{i} \rightarrow 0 ; q_{i} \rightarrow 0\right)$.

The simplest isotherm model is the single-site Langmuir isotherm

$$
c_{i}=c_{i, \mathrm{sat}} \frac{b_{i} f_{i}}{1+b_{i} f_{i}} ; \theta_{i}=\frac{b_{i} f_{i}}{1+b_{i} f_{i}} ; \quad b_{i} f_{i}=\frac{\theta_{i}}{1-\theta_{i}}
$$

where we define the fractional occupancy of the adsorbate molecules, $\theta_{i}$

$$
\theta_{i}=c_{i} / c_{i, \mathrm{sat}}=q_{i} / q_{i, \mathrm{sat}}=\Theta_{i} / \Theta_{i, \mathrm{sat}}
$$

Differentiation of eqn (7) yields the following expression for $\Gamma_{i}$

$$
\Gamma_{i}=1 /\left(1-\theta_{i}\right)
$$

Many of the complex $D_{i} v s . c_{i}$ dependencies of practical interest require more detailed descriptions of adsorption equilibrium than afforded by eqn (7). It is sufficient for the purposes of this article to consider two extensions of the single-site Langmuir model. As an example, for a proper interpretation of the curious maximum $D_{i} v s$. $c_{i}$ observed for $\mathrm{CH}_{4} / \mathrm{LTA}-5 \mathrm{~A}(c f$. Fig. 3c), 
(a)

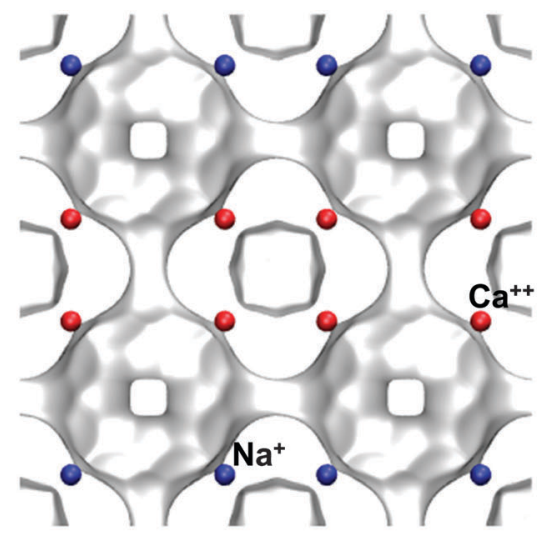

(b)

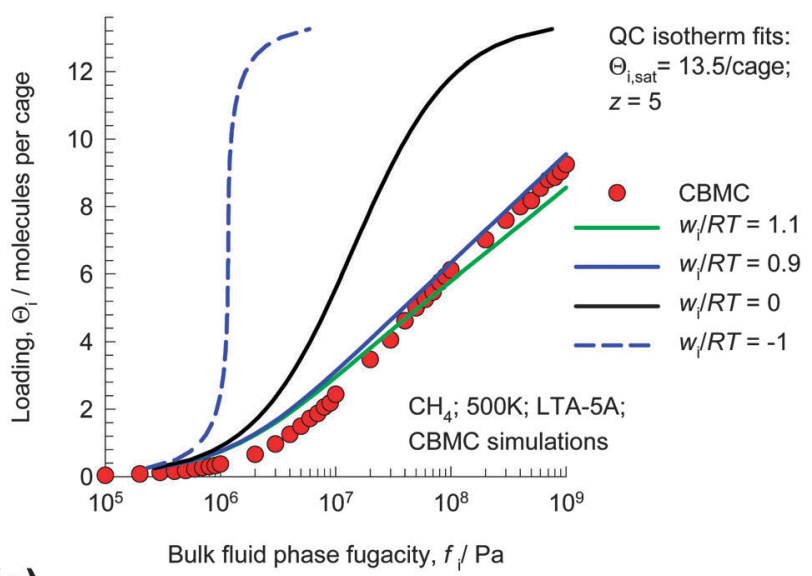

(c)

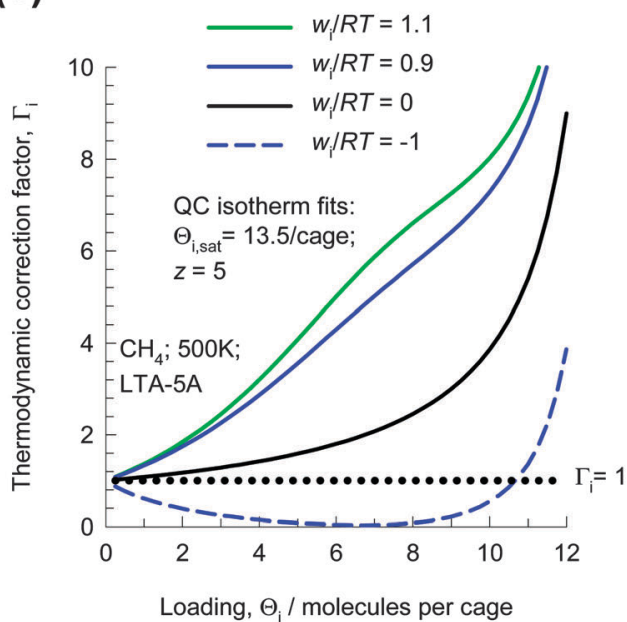

Fig. 4 (a) Pore landscape of the LTA-5A zeolite. (b) CBMC simulations of the $\mathrm{CH}_{4}$ isotherm in LTA-5A at $500 \mathrm{~K}$, compared with the calculations of the quasichemical isotherm model with four different $\left(w_{i} / R T\right)$ values. (c) Comparison of the thermodynamic correction factors $\Gamma_{i}$ for $\mathrm{CH}_{4}$ in LTA-5A for four different $\left(w_{i} / R T\right)$ values.

typical of cage-type zeolites with 8-ring windows, we need to take explicit account of adsorbate-adsorbate interactions. Application of the quasi-chemical (QC) mean field approximation of
Guggenheim for adsorption on a lattice with a uniform coordination number, $z$, with nearest neighbor interaction energies, $w_{i}$, yields ${ }^{1,36}$

$$
b_{i} f_{i}=\frac{\theta_{i}}{\left(1-\theta_{i}\right)}\left(\frac{2\left(1-\theta_{i}\right)}{s_{i}+1-2 \theta_{i}}\right)^{z}
$$

where we define

$$
\varsigma_{i}=\sqrt{1-4 \theta_{i}\left(1-\theta_{i}\right)\left(1-\exp \left(-w_{i} / R T\right)\right)}
$$

Differentiation of eqn (10) yields the remarkably simple expression for the thermodynamic factor ${ }^{37,38}$

$$
\Gamma_{i}=\frac{1}{\left(1-\theta_{i}\right)}\left(1+\frac{z}{2} \frac{\left(1-\varsigma_{i}\right)}{\varsigma_{i}}\right)
$$

For a square lattice, the coordination number $z=4$; for a cubic lattice, $z=6$. Some guidelines for the determination of $z$ for zeolites and MOFs are obtained by setting up a Kinetic Monte Carlo scheme for molecular hopping. ${ }^{39,40}$ In the limiting case of negligible adsorbate-adsorbate interactions, $w_{i}=0 ; \zeta_{i}=1$, eqn (10) and (12) reduce to eqn (7), and (9), respectively.

The influence of the molecule-molecule interactions becomes increasingly significant as the loading increases. In order to demonstrate this, CBMC simulations of the $\mathrm{CH}_{4}$ isotherm in LTA-5A are compared with the calculations of the QC model taking $\left(w_{i} / R T\right)=0,0.9,1.1$, and -1.0 ; see Fig. $4 \mathrm{~b}$. The isotherms become increasingly steep as $\left(w_{i} / R T\right)$ decreases. A negative value of the interaction implies that intermolecular interactions are attractive in nature, and in this scenario yields a near-vertical isotherm for $\left(w_{i} / R T\right)=-1.0$. Steep isotherms are typically observed for $n \mathrm{C}_{4} \mathrm{H}_{10}$ and $n \mathrm{C}_{6} \mathrm{H}_{14}$ in some MOFs see Fig. $1 \mathrm{~d}$ and e. In principle it is possible to fit the isotherm data to determine both $b_{i}$ and $\left(w_{i} / R T\right)$. Attractive interactions often lead to molecular clustering over a range of concentrations, and this occurrence is best illustrated by calculations of the thermodynamic factor $\Gamma_{i}$ in Fig. 4c. For $\left(w_{i} / R T\right)=-1.0$, we note that $\Gamma_{i}<1$ for a range of loadings, resulting in the hierarchy of diffusivities $D_{i \text {,self }} \leq \bigoplus_{i} \geq D_{i}$.

The uniform distribution of adsorption sites assumed in the derivation of eqn (10) is not applicable in cases such as that occurring with strong binding of $\mathrm{CO}_{2}$, alkenes, and alkynes to the metal atoms of MgMOF-74 (ref. 41) and FeMOF-74 (ref. 5) frameworks. This is best illustrated by the experimental isotherm for $\mathrm{CO}_{2}, \mathrm{C}_{2} \mathrm{H}_{2}, \mathrm{C}_{2} \mathrm{H}_{4}$, and $\mathrm{C}_{3} \mathrm{H}_{6}$ adsorption in MgMOF-74; see Fig. 5a. There are $18 \mathrm{Mg}$ atoms in a unit cell of MgMOF-74, and a maximum loading of 18 per unit cell is achievable at these strong binding sites. Higher loadings are achievable by location at other positions in the metal framework with weaker binding. Consequently, a pronounced inflection is observed at a loading $\Theta_{i}=18$. Such strong inflection characteristics require fitting with the dual-Langmuir-Freundlich model ${ }^{9,42}$

$$
c_{i}=c_{i, \mathrm{~A}, \mathrm{sat}} \frac{b_{i, \mathrm{~A}} f_{i}^{\nu_{i, \mathrm{~A}}}}{1+b_{i, \mathrm{~A}} f_{i}^{\nu_{i, \mathrm{~A}}}}+c_{i, \mathrm{~B}, \mathrm{sat}} \frac{b_{i, \mathrm{~B}} f_{i}^{\nu_{i, \mathrm{~B}}}}{1+b_{i, \mathrm{~B}} f_{i}^{\nu_{i, \mathrm{~B}}}}
$$

The strong binding energies of $\mathrm{CO}_{2}, \mathrm{C}_{2} \mathrm{H}_{2}, \mathrm{C}_{2} \mathrm{H}_{4}$, and $\mathrm{C}_{3} \mathrm{H}_{6}$ are underscored by the high values of the isosteric heats 
(a)

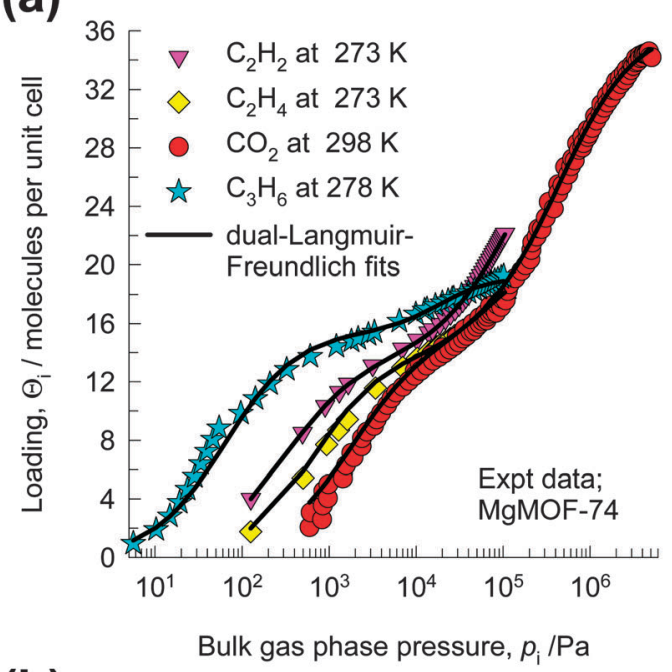

(b)

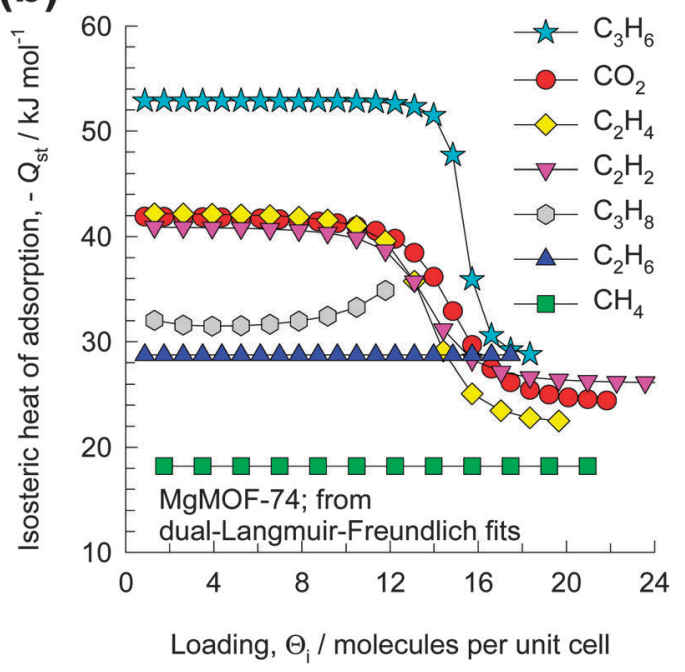

Fig. 5 Experimental data ${ }^{9}$ for (a) adsorption isotherms, and (b) isosteric heats of adsorption, $-\mathrm{Q}_{\text {st, }}$ for $\mathrm{CO}_{2}, \mathrm{CH}_{4}, \mathrm{C}_{2} \mathrm{H}_{2}, \mathrm{C}_{2} \mathrm{H}_{4}, \mathrm{C}_{2} \mathrm{H}_{6}, \mathrm{C}_{3} \mathrm{H}_{6}$, and $\mathrm{C}_{3} \mathrm{H}_{8}$ in MgMOF-74.

of adsorption, $-Q_{\mathrm{st}}$; see Fig. $5 \mathrm{~b}$. The values of $-Q_{\mathrm{st}}$ are significantly lower when $\Theta_{i}>18$.

Steep isotherms and molecular clustering phenomena are adequately captured with eqn (13) by taking at least one of the exponents, $\nu_{i}$, to have values exceeding unity. Fig. 3 also includes the data for Fick $D_{i}$ for $\mathrm{CH}_{4}$ diffusion, calculated using the appropriate isotherm fits using eqn (13). For all six host materials, $1 / \Gamma_{i} \leq 1$, and consequently the hierarchy of diffusivities is $D_{i, \text { self }} \leq D_{i} \leq D_{i}$ over the entire concentration range. In the limit of vanishing small pore concentrations or molar loadings, $c_{i} \rightarrow 0 ; q_{i} \rightarrow 0$, all three diffusivities converge to the same value; this limiting value is termed the "zero loading" diffusivity and is denoted $D_{i}(0)$. Except for the mesoporous host BTP-COF, we note that the concentration dependence of the Fick diffusivity $D_{i}$ exhibits inflection characteristics; this is a direct consequence of the inflections in the corresponding isotherms. The MD data in Fig. 3 clearly show that the "corrected" diffusivity $\emptyset_{i}$ is not concentration independent.
We now proceed to elucidate how the various factors such as $\Gamma_{i},\left(w_{i} / R T\right)$, and $-Q_{\mathrm{st}}$ leave strong imprints on the magnitudes and concentration dependencies of diffusivities.

\section{Influence of adsorbate-adsorbate interactions on intercage hopping in cage-type structures}

Zeolite structures such as LTA-5A, CHA, DDR, ITQ-29, and ERI consist of cages separated by 8-ring windows in the 3.2-4.2 size range. In such structures, guest molecules jump one-at-atime across the windows. As a first approximation, the intercage hopping of molecules can be considered to be uncorrelated, i.e. $D_{i, \text { self }} \approx D_{i},{ }^{17}$ as witnessed in Fig. $3 \mathrm{c}$ with data for $\mathrm{CH}_{4}$ diffusivities in LTA-5A. The constraining window regions offer significant free energy barriers for inter-cage hopping of molecules. It is clear from Fig. 4b that the CBMC simulated isotherms for LTA-5A can only be matched if repulsive adsorbate-adsorbate interactions are accounted for with $\left(w_{i} / R T\right) \approx 1$. An important consequence of this is that the free energy of the molecules within the cage increases with increasing cage occupancy; this is demonstrated by the molecular simulations of the free energy profiles; see Fig. 6a. The free energy within the cage is significantly higher at a loading of 6 per cage when compared to the values for 1 per cage. It is also noteworthy that the free energy at the window regions is practically independent of the loading. The free energy barrier for inter-cage hopping, $\delta F_{i}$, may be defined as the difference between the values of free energy at the window region and within the cage; $\delta F_{i}$ decreases with increased loading. A consequence of the reduction of $\delta F_{i}$ with increase in $\Theta_{i}$ is that $D_{i \text {,self }}$ increases with $\Theta_{i}$ in the range $0<\Theta_{i}<9$; see MD simulation data in Fig. 6b. The increase of $D_{i}$ with $\Theta_{i}$ is not monotonic because the cage capacity is limited and there are fewer intra-cage vacant sites to occupy. As the saturation loading is approached, progressively fewer vacant sites become available, $\left(1-\theta_{i}\right) \rightarrow 0$. The net result is that $D_{i, \text { self }}$ displays a maximum at $\Theta_{i} \approx 9$, and decreases thereafter.

A detailed analysis of the influence of free energy barriers on the diffusivity in cage-type zeolites is available in the papers by Beerdsen et al. ${ }^{43,44}$ Here, we proceed further using the quasichemical model to quantify the loading dependencies based on the assumption that the hopping frequencies of molecules between cages are influenced by a factor $\exp \left(w_{i} / R T\right)$, leading to the following explicit expression ${ }^{1,37-39}$

$$
D_{i}=D_{i}(0)\left(\frac{1+\varsigma_{i}}{2\left(1-\theta_{i}\right)}\right)^{-z}\left(1+\frac{\left(\varsigma_{i}-1+2 \theta_{i}\right) \exp \left(w_{i} / R T\right)}{2\left(1-\theta_{i}\right)}\right)^{z-1}
$$

The continuous solid lines in Fig. $6 \mathrm{~b}$ are calculations using eqn (14), taking $\left(w_{i} / R T\right)=0,0.9$ and 1.1 , signifying repulsive interactions. Increasing the degree of repulsive interactions between adsorbates has the effect of increasing the free-energies within the cage. We see that the choice of $\left(w_{i} / R T\right)=1.1$ that matches the isotherm data ( $c f$. Fig. $4 \mathrm{a}$ ) is capable of capturing the essential features of the loading dependence determined 
(a)

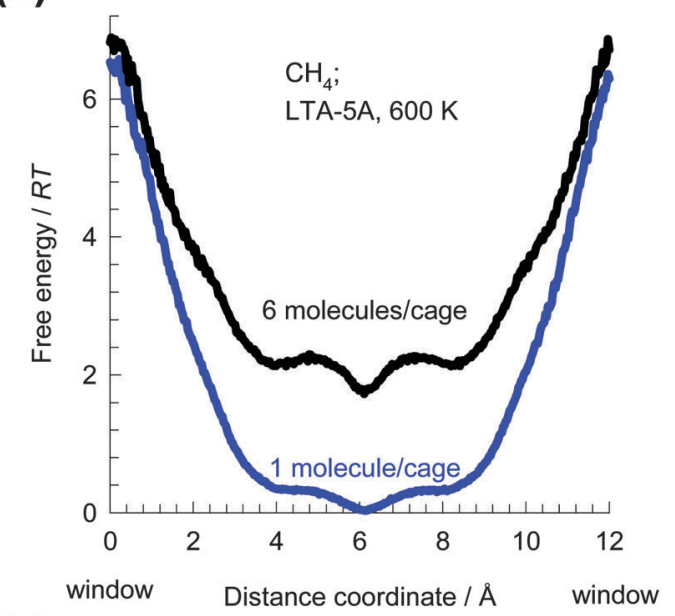

(b)

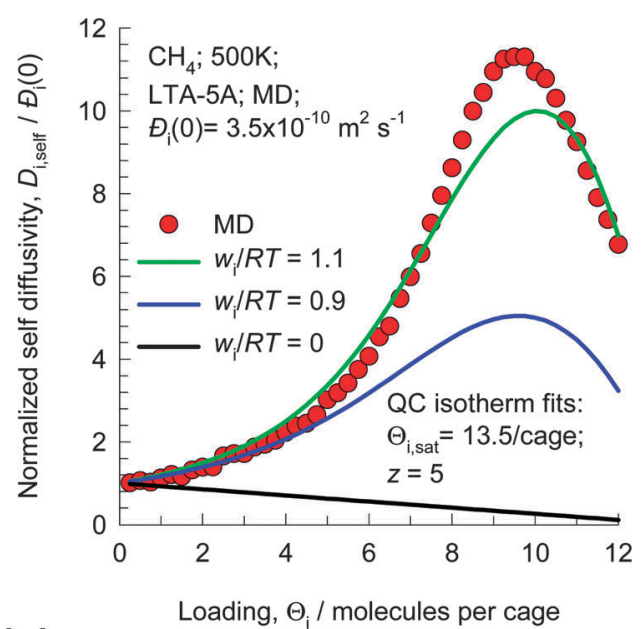

(c)

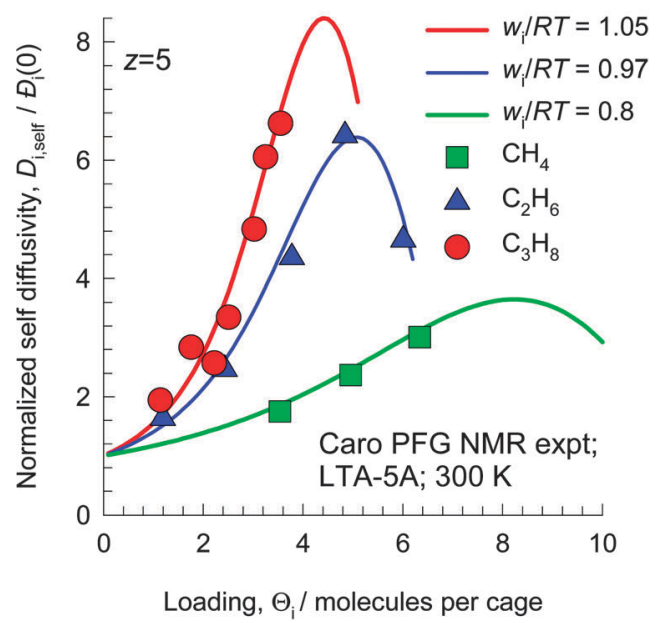

Fig. 6 (a) Free energy profiles for $\mathrm{CH}_{4}$ in LTA-5A, determined by molecular simulations for loadings of 1 and 6 molecules per cage. ${ }^{20}(\mathrm{~b})$ Comparison of MD simulations of the self-diffusivities, $D_{i, \text { self, }}$ normalized with respect to the zero-loading value $E_{i}(0)$, of $\mathrm{CH}_{4}$ in LTA-5A at $500 \mathrm{~K}$ with calculations using eqn (14), taking $\left(W_{i} / R T\right)=0,0.9$ and 1.1. (c) PFG NMR experimental data of Caro et al. ${ }^{45}$ for diffusivities of $\mathrm{CH}_{4}, \mathrm{C}_{2} \mathrm{H}_{6}$, and $\mathrm{C}_{3} \mathrm{H}_{8}$ in the LTA-5A zeolite at $300 \mathrm{~K}$. The experimental data have been normalized taking the zero-loading diffusivity values, $D_{i}(0)=1 \times 10^{-9}, 6 \times 10^{-11}$, and $1.75 \times$ $10^{-13} \mathrm{~m}^{2} \mathrm{~s}^{-1}$, respectively, the continuous solid lines are calculations using eqn (14). from MD. The assumption of negligible adsorbate-adsorbate interactions, $\left(w_{i} / R T\right)=0$, results in a linear dependence

$$
\bigoplus_{i}=\bigoplus_{i}(0)\left(1-\theta_{i}\right) ;\left(w_{i} / R T\right)=0
$$

The PFG NMR experimental data of Caro et al. ${ }^{45}$ for selfdiffusivities in LTA-5A can be modelled reasonably well by taking $\left(w_{i} / R T\right)=0.8,0.97$ and 1.05 for methane, ethane, and propane, respectively; see Fig. 6c.

The cage capacities of LTA, CHA, ITQ-29, and DDR are not the same; the values are determined as LTA-5A $=702 \AA^{3}$; ITQ-29 = $677 \AA^{3}$; CHA $=316 \AA^{3} ;$ DDR $=363 \AA^{3}$; ERI $=408 \AA^{3}$; AFX $=588 \AA^{3}$; ZIF-8 $=1168 \AA^{3}$. As a consequence the loading at which the maximum in the diffusivity is attained is different for each structure as illustrated by data in Fig. 7a. The cage capacities of CHA and DDR are the smallest, and therefore the peaks in the diffusivity values manifest at lower cage loadings. For each structure eqn (14) provides a good description of the loading dependence, with values of $\left(w_{i} / R T\right)$ describing the adsorption isotherms; see ESI $\dagger$ for details.

The Fick diffusivity $D_{i}$, which is the product of the M-S diffusivity $\bigoplus_{i}$ and the thermodynamic correction factor $\Gamma_{i}$, increases even with loading more strongly than $\emptyset_{i}$ ( $c f$. Fig. 3c). This has important consequences for transient uptake within crystalline materials. Experimental data of Garg and Ruthven ${ }^{46}$ for uptake of $\mathrm{C}_{2} \mathrm{H}_{6}$ in LTA-4A show that the adsorption cycle equilibrates significantly faster than the desorption phase; see Fig. 7b. The asymmetry in the adsorption and desorption rates is a direct consequence of the strong loading dependence of $D_{i}$. In the adsorption cycle, the progressive loading increase during uptake has an "acceleration" effect. Conversely, in the desorption cycle there is "deceleration" due to progressive reduction in the loading. The proper modelling of adsorption-desorption transience requires us to take due account of the loading dependencies. ${ }^{47}$

The zero-loading diffusivities $\bigoplus_{i}(0)$ are strongly dependent on the window dimensions, and a reduction of $0.5 \AA$ in size of the window aperture can lead to two orders of magnitude decrease in magnitude; see Fig. 7c. ${ }^{48,49}$ For separations of propene-propane mixtures, of importance in the production of polymer grade propene, diffusion selectivities $>1000$ in favour of the unsaturated propene can be achieved with $\mathrm{CHA}$ and DDR zeolites; ${ }^{50}$ see Fig. 7d. The differences in the diffusivities are due to subtle differences in the bond lengths and bond angles of propene and propane. The commonly used kinetic diameters of molecules are not the proper determinants of the diffusivities of pencil or banana shaped molecules. Pencil shaped $\mathrm{CO}_{2}$ and $\mathrm{N}_{2}$ molecules jump length-wise across the windows of CHA, DDR, LTA and have diffusivities that are about one to three orders of magnitude higher than that of the spherical $\mathrm{CH}_{4}$ molecule. For this reason, CHA, DDR, and LTA membranes are attractive for use in membrane separations of $\mathrm{CO}_{2}-\mathrm{CH}_{4}$ and $\mathrm{CH}_{4}-\mathrm{N}_{2}$ mixtures. ${ }^{27,28,51}$

Lattice flexibility has little influence on the inter-cage hopping across narrow 8-ring windows of cage-type zeolites. ${ }^{48,49}$ The flexibility of the windows of ZIF-8 has distinctively different characteristics causing the effective aperture size to be higher than the crystallographic value of $3.26 \AA$. For example, MD simulations 
(a)

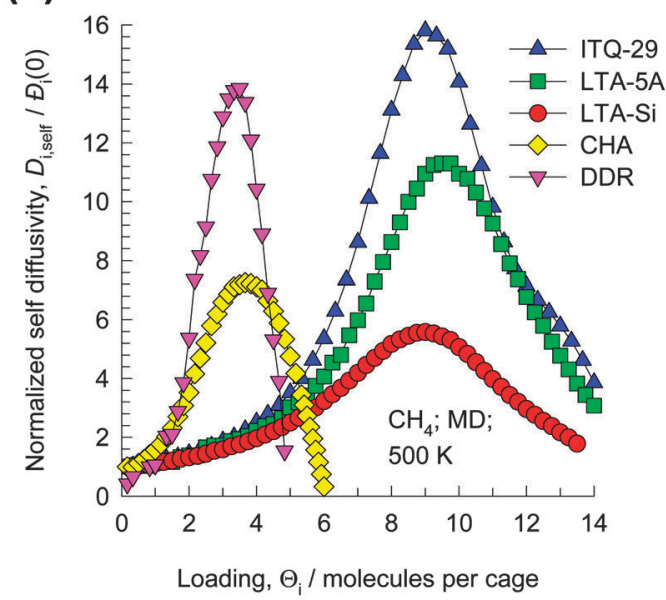

(c)

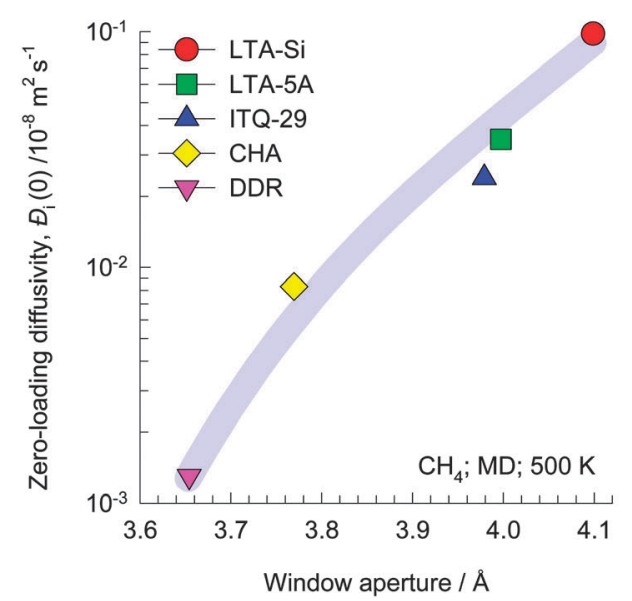

(b)

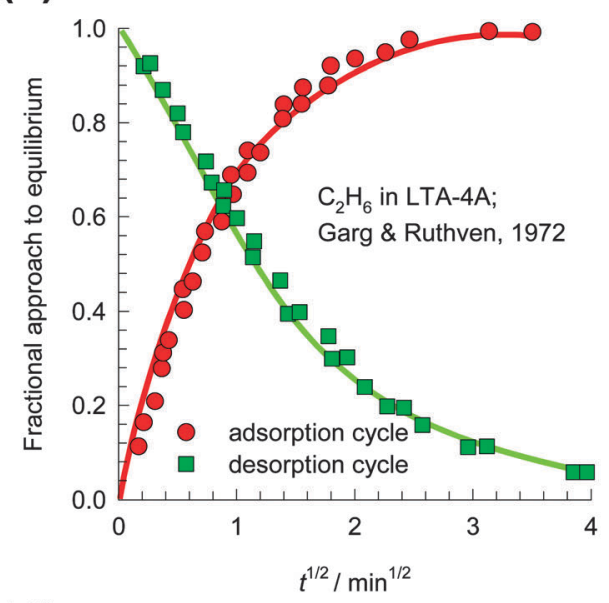

(d)

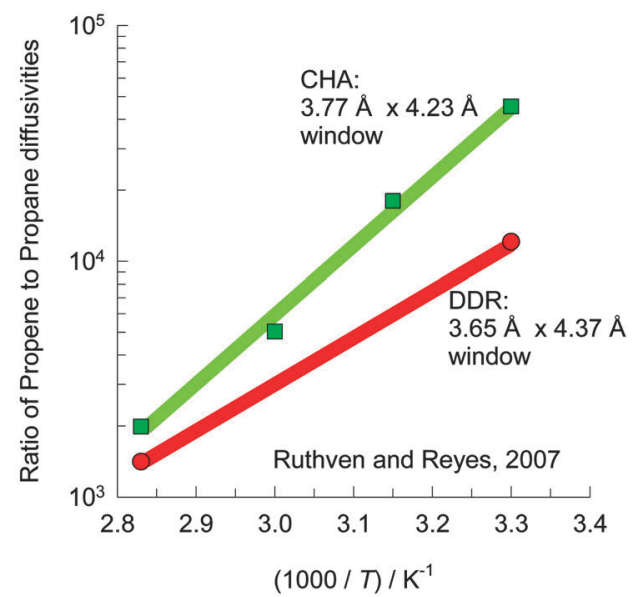

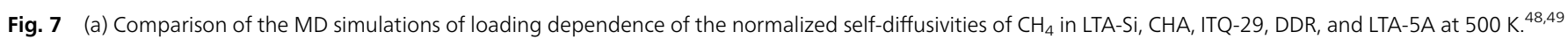

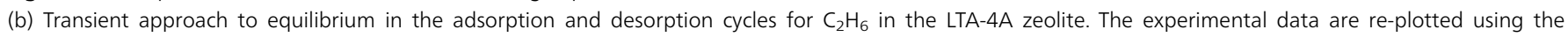

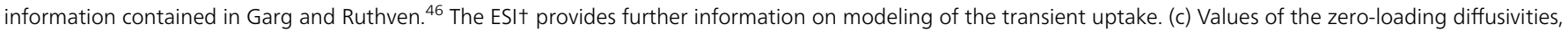

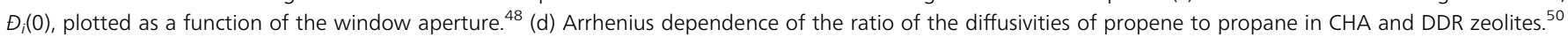

of the diffusivity of $\mathrm{CH}_{4}$ in ZIF-8 are found to be about two orders of magnitude higher when framework flexibility effects are accounted for. ${ }^{52}$ Framework flexibility of ZIF-8 also explains why molecules as bulky as 2,2 dimethylbutane can hop across the windows. ${ }^{13,53,54}$ From uptake measurements, Li et al. ${ }^{55}$ have determined the ratio of diffusivities of propene and propane in ZIF-8 to be 125, significantly lower than the values obtained for CHA and DDR that have larger window apertures; the framework flexibility of ZIF-8 is a plausible explanation for this anomaly.

\section{Commensurateness of molecular dimensions with periodicity of framework structure}

There is an inherent periodicity in the pore landscape of nanoporous crystalline structures. For diffusion of homologous series of chain molecules, such as alkanes and alcohols, there could be either a match, or mis-match, between the characteristic periodicity of the structure and the characteristic length of the guest molecules. The net result could be a non-monotonic variation of the diffusivity with chain length. This non-monotonicity has been given a variety of names in the literature: resonant diffusion, ${ }^{23,56,57}$ window effect, ${ }^{58}$ and commensurateincommensurate diffusion. ${ }^{59-61}$

The periodic nature is best illustrated by pore landscapes such as for LTA-5A, CHA, ERI, DDR, and AFX in Fig. 8. This periodicity is dictated by the window-to-window distance, and the cage dimensions. For adsorption of linear chain molecules such as $n$-alkanes and $n$-alcohols, the number of molecules that can be located within each cage decreases with increasing chain length. This is illustrated by the snapshots in Fig. 9 showing the conformations and locations of linear alkanes in CHA. The saturation loadings are $\mathrm{C} 1=6 ; \mathrm{C} 2=5$; $\mathrm{C} 3=4 ; n \mathrm{C} 4=$ $n \mathrm{C} 5=2 ; n \mathrm{C} 6=n \mathrm{C} 7=n \mathrm{C} 8=1$ molecules per cage. For longer chains such as $n \mathrm{C} 12$, the capacity of a single CHA cage is too limited and the linear molecule has to straddle two adjacent cages. The molecular dimension of $n \mathrm{C} 12$ is incommensurate 

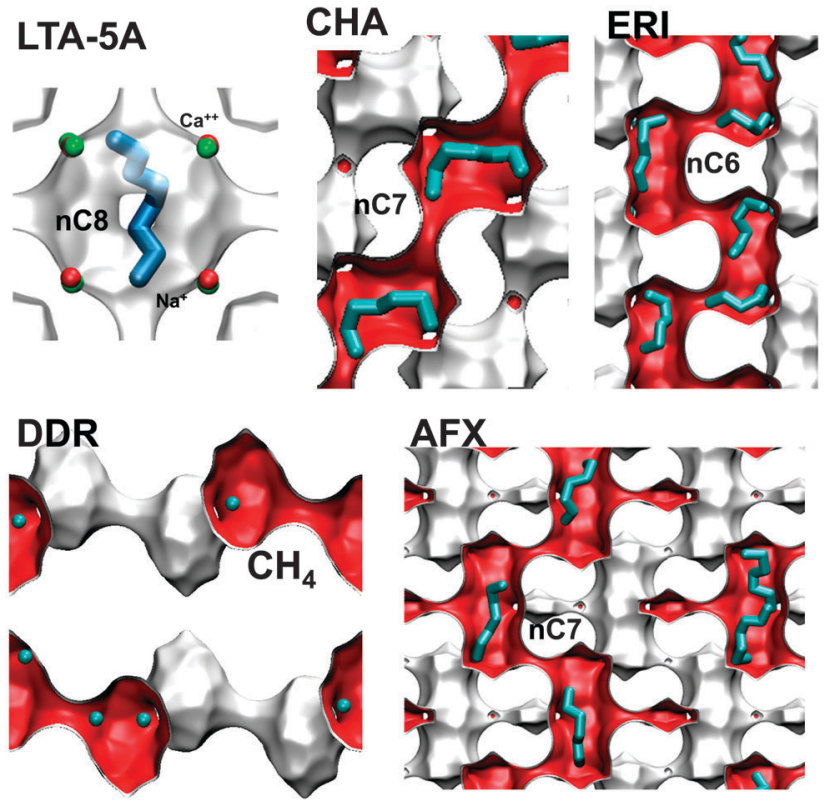

Fig. 8 Pore landscapes of LTA-5A, CHA, ERI, DDR, and AFX. Also shown are the snapshots, respectively, of the adsorbed molecules $n C 8, n C 7, n C 6, \mathrm{CH}_{4}$, and $n C 7$.
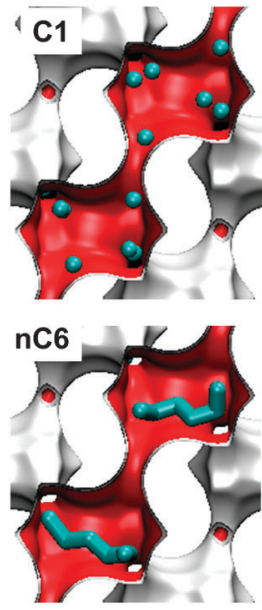

Fig. 9 Snapshots showing the location of $\mathrm{C} 1, \mathrm{C} 2, n \mathrm{C} 4, n \mathrm{C} 7, n \mathrm{C} 8$, and $n \mathrm{C} 12$ alkane molecules within the cages of CHA.

with the periodicity of the CHA pore landscape; this has consequences for its diffusion characteristics.

Dubbeldam et al. ${ }^{59-61}$ have carried out a detailed investigation of the consequences of commensurate-incommensurate scenarios on adsorption and diffusion of linear alkanes in cagetype zeolites with the aid of rare event simulations. The simulation data demonstrate that the adsorption and diffusion characteristics exhibit non-monotonic variation with increasing chain length, illustrated by the data for Henry coefficients and zero-loading diffusivities of $n$-alkanes in CHA in Fig. 10a. The chain-length dependence of the diffusivities is nearly the mirror-image of the corresponding variation of the adsorption strengths, quantified in this case by the Henry coefficient. An incommensurate scenario leads to a higher diffusivity because (a)

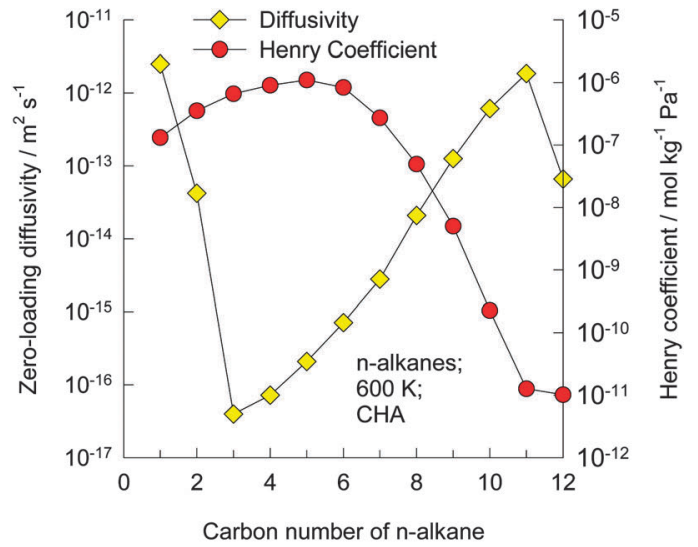

(b)

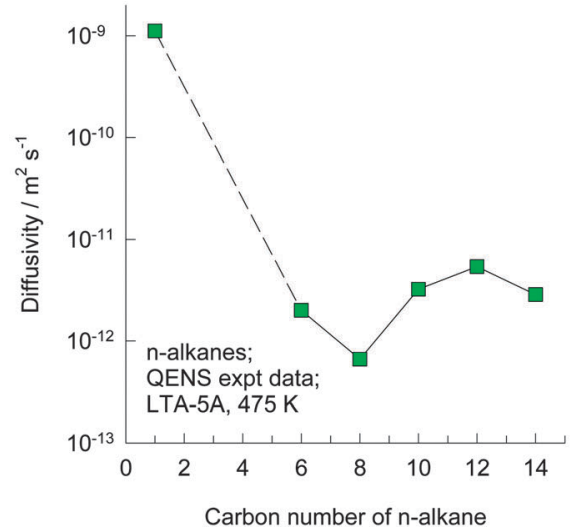

Fig. 10 (a) Molecular simulation data for the Henry coefficient and zero-loading diffusivity of linear alkanes in the CHA zeolite at $600 \mathrm{~K}^{61}$ (b) QENS data of Jobic et al. ${ }^{62}$ for diffusivity of $n$-alkanes in LTA-5A.

the molecule does not feel "too comfortable" and has a propensity to be more mobile; this is the rationale why the diffusivities of $n \mathrm{C} 11$ and $n \mathrm{C} 12$ are higher than those of $n \mathrm{C} 6$, $n \mathrm{C} 7$, and $n \mathrm{C} 8$.

The QENS data of Jobic et al. ${ }^{62}$ for diffusivities in LTA-5A with increasing $n$-alkane chain length display a minimum value for $n$ C8; see Fig. 10b. $n$ C8 can nestle comfortably within a single cage, as evidenced in the snapshot in Fig. 8. In sharp contrast, the dimensions of $n \mathrm{C} 13$ are too long to fit into one cage, i.e. incommensurate with the window-to-window distance, and straddle two adjacent cages. This rationalizes the experimental observation that $n \mathrm{C} 13$ has a higher diffusivity than $n \mathrm{C} 8$.

For intersecting channel structures such as MFI, ISV, and BOG the framework periodicity is introduced by the distances between adjacent channel intersections. The length of an $n \mathrm{C} 6$ alkane molecule is commensurate with the distance between intersections of the MFI zeolite ( $c f$. snapshot in Fig. 11a), providing a rationale for the experimental data obtained from membrane permeation showing a minimum in the diffusivity of $n \mathrm{C} 6$; $c f$. Fig. 11b. Longer $n \mathrm{C} 7$ and $n \mathrm{C} 8$ chains have incommensurate lengths and exhibit higher diffusivities than $n$ C6. 
(a)

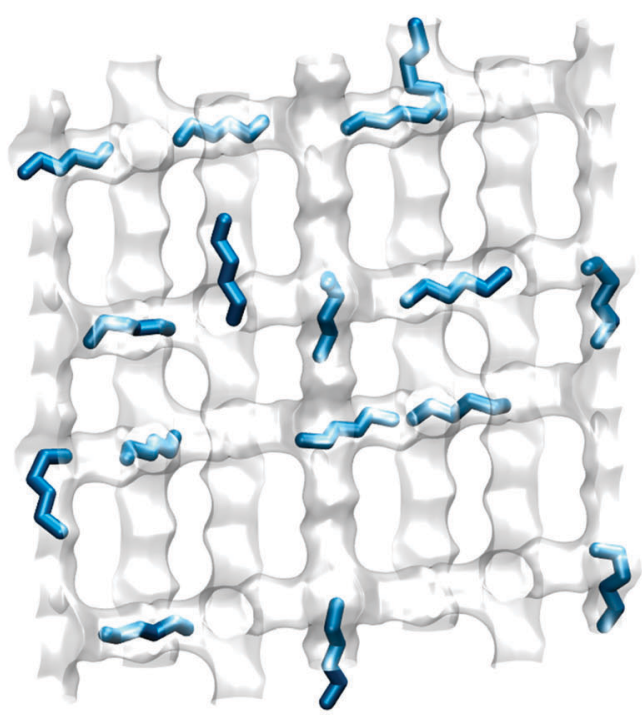

(b)

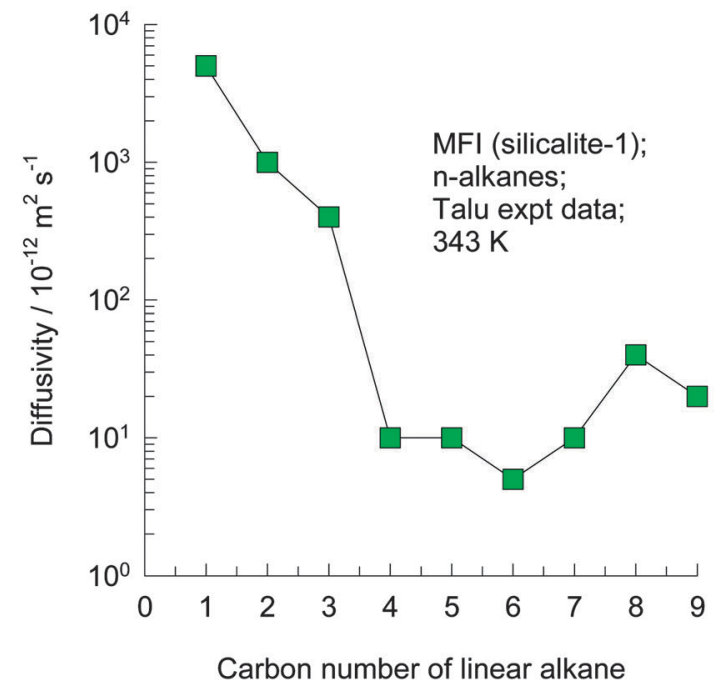

Fig. 11 (a) Snapshot showing the location of $n C 6$ in MFI at a loading of 4 molecules per unit cell. (b) Diffusivities of linear alkanes in the MFI zeolite obtained from membrane permeation experiments. ${ }^{93}$

For one-dimensional (1D) channel structures such as AFI, LTL, TON, MTW, Co-FA, and Mn-FA, the periodicity is introduced

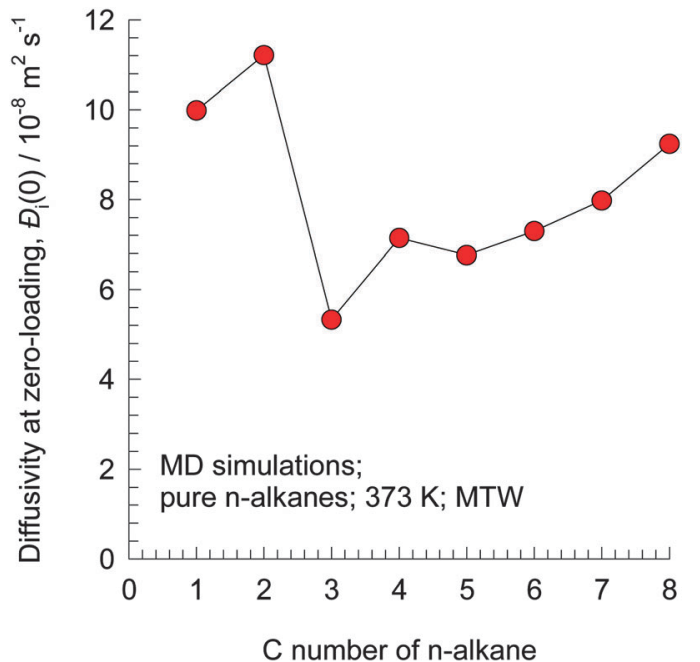

Fig. 13 MD simulation data for the zero-loading diffusivities, $\Theta_{i}(0)$, of $n$-alkanes in MTW at $373 \mathrm{~K}^{20}$

by the segment length of wall corrugations. For adsorption of linear alkanes, there is either a subtle match, or mis-match, between the molecular dimensions and the segment length. This is illustrated in Fig. 12 by the snapshots of linear alkanes within the $1 \mathrm{D}$ channels of the MTW zeolite. Fig. 13 shows MD simulation data for the zero-loading diffusivities of $n$-alkanes in MTW showing a minimum value for propane; the most likely reason is that the length of $\mathrm{C} 3$ is commensurate with the segment length. Molecular simulations have also demonstrated that the diffusivities of linear alkanes in Co-FA and Mn-FA have a non-monotonic character. ${ }^{63}$

\section{Influence of binding energy on zero-loading diffusivities $\emptyset_{i}(0)$}

For diffusion in microporous structures with characteristic channel dimensions larger than say $5 \AA$, there are often no discernible regions that afford high energy barriers to molecular jumps. The $\bigoplus_{i}(0)$ is influenced by a wide variety of factors such as molecular dimensions, molecular conformations, and binding energies. ${ }^{64}$ Let us now investigate the extent to which the high binding energies of $\mathrm{CO}_{2}$ and alkenes serve to reduce

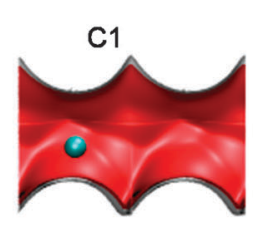

$\mathrm{nC6}$

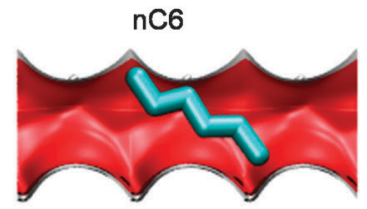

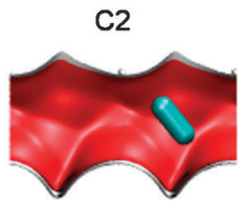

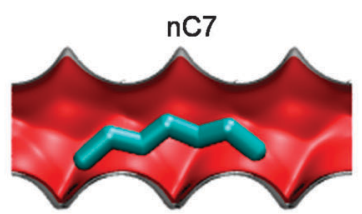

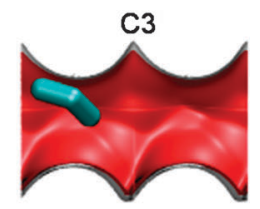

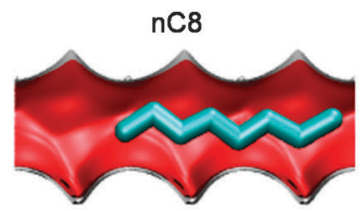

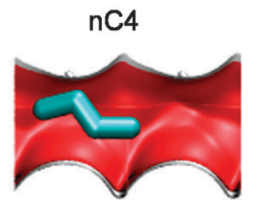

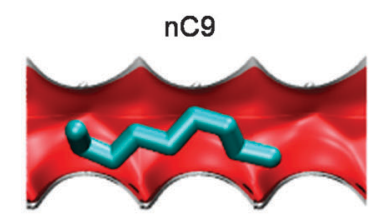

Fig. 12 Snapshots showing the conformation of linear alkanes within the 1D channels of MTW. 
(a)

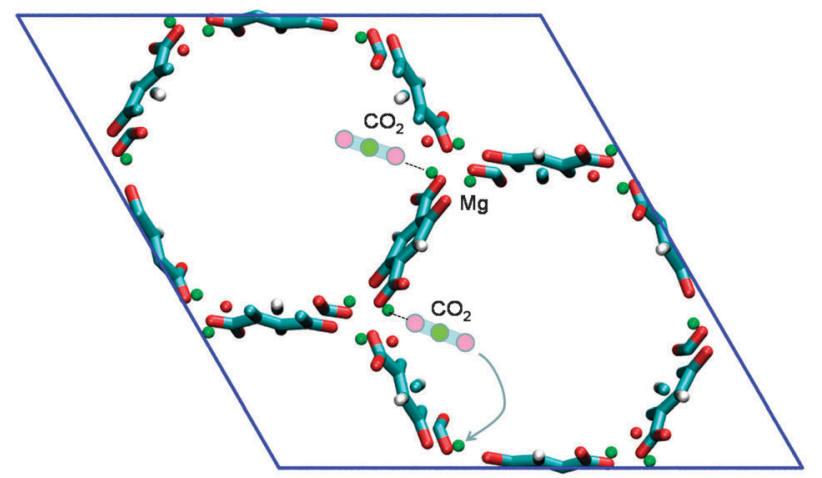

(b)

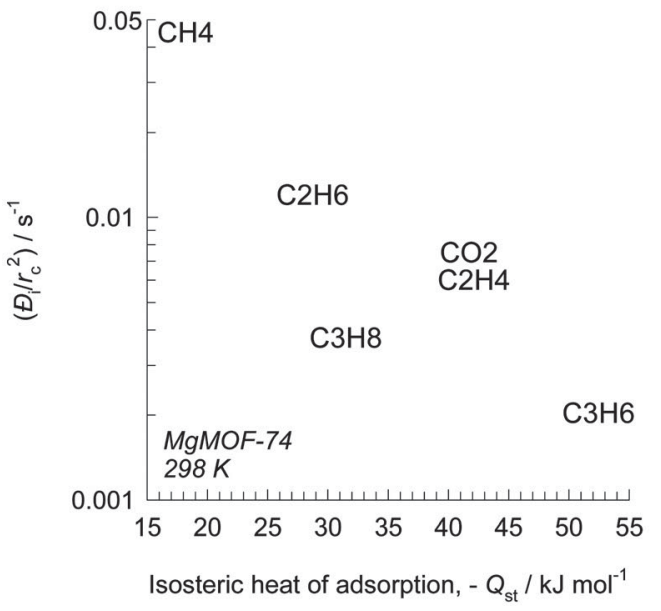

(c)

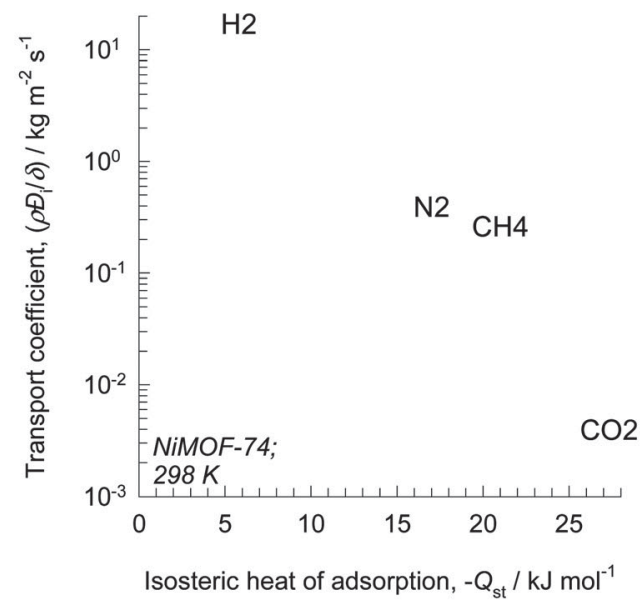

Fig. 14 (a) Snapshot showing the location of adsorbed $\mathrm{CO}_{2}$ molecules within the 1D hexagonal-shaped channels of MgMOF-74. (b) The values of ${D_{i}}_{i} / r_{c}^{2}$ for $\mathrm{CO}_{2}, \mathrm{CH}_{4}, \mathrm{C}_{2} \mathrm{H}_{4}, \mathrm{C}_{2} \mathrm{H}_{6}, \mathrm{C}_{3} \mathrm{H}_{6}$, and $\mathrm{C}_{3} \mathrm{H}_{8}$ in MgMOF-74 plotted as a function of $-Q_{\text {st. }}{ }^{64}$ (c) The membrane transport coefficient, $\rho D_{i} / \delta$, of $\mathrm{H}_{2}, \mathrm{~N}_{2}, \mathrm{CH}_{4}, \mathrm{CO}_{2}$ in NiMOF-74 as a function of $-Q_{\text {st. }}{ }^{64}$

the corresponding diffusivities within the 1D hexagonal shaped channels of MgMOF-74. Neutron diffraction data ${ }^{41}$ establish (a)

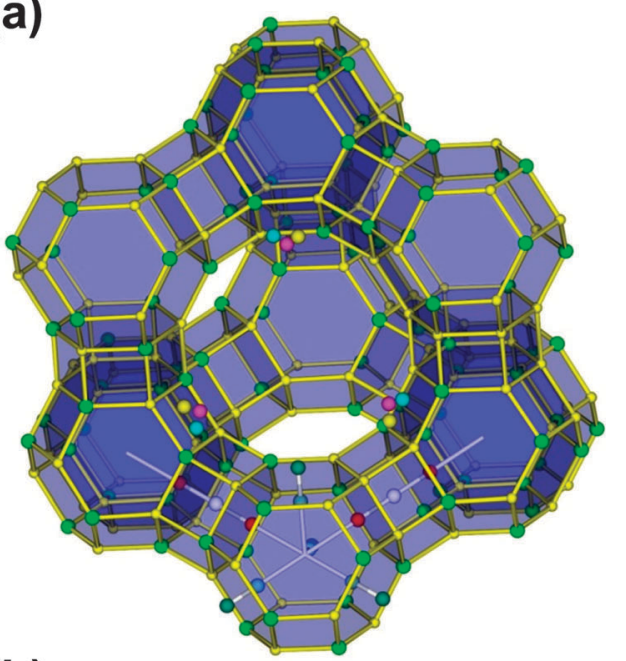

(b)

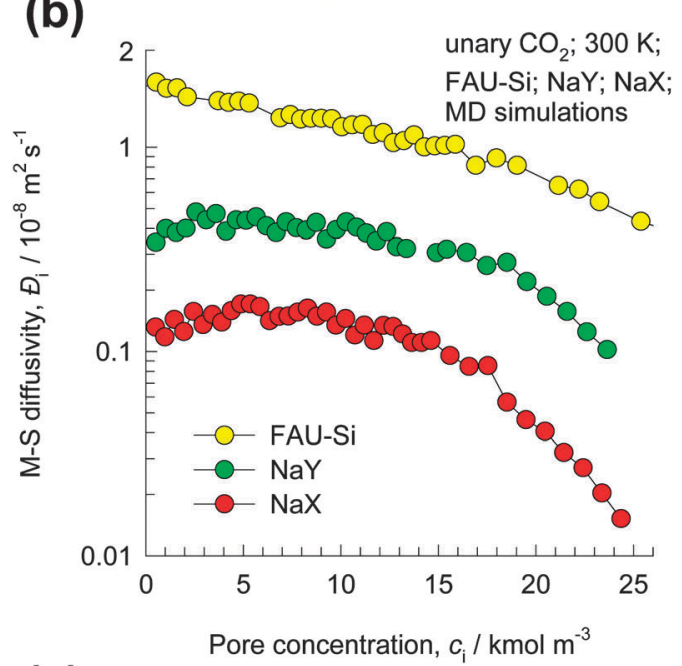

(c)

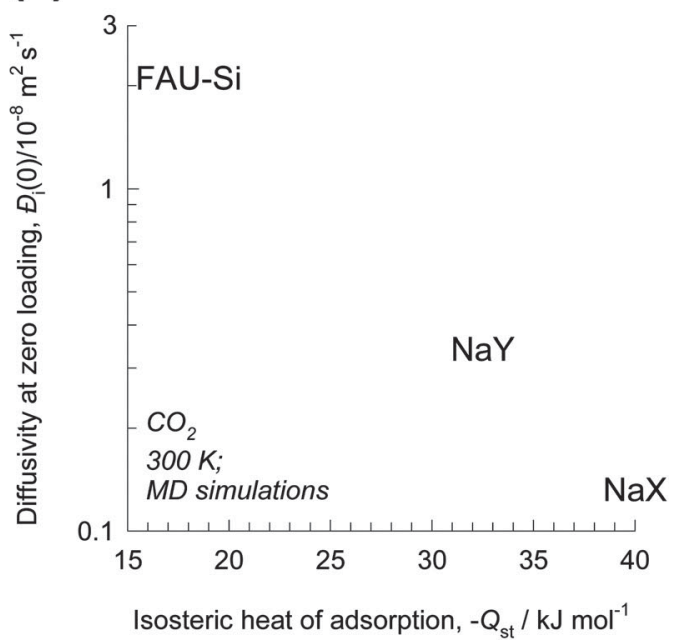

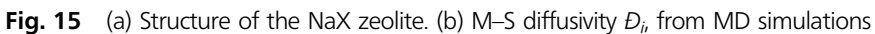
for pure $\mathrm{CO}_{2}$ in FAU-Si (all-silica), $\mathrm{NaY}\left(54 \mathrm{Na}^{+} ; \mathrm{Si} / \mathrm{Al}=3\right)$, and $\mathrm{NaX}\left(86 \mathrm{Na}^{+}\right.$; $\left.\mathrm{Si} / \mathrm{Al}=1.23\right)$, as a function of the pore concentration $c_{i}{ }^{21}$ (c) Plot of the zero-loading diffusivities $\Theta_{i}(0)$ versus the corresponding values of the isosteric heats of adsorption, $-Q_{\text {st }}$.

that $\mathrm{CO}_{2}$ molecules attach via $\mathrm{O}$ atoms to the $\mathrm{Mg}$ atoms of the framework as pictured in Fig. 14a. Intuitively one might expect 
(a)

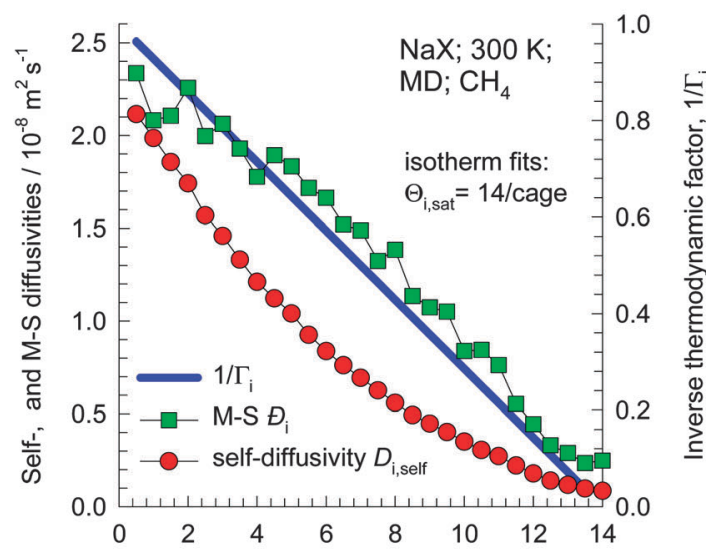

(b) Loading, $\Theta_{\mathrm{i}} /$ molecules per cage

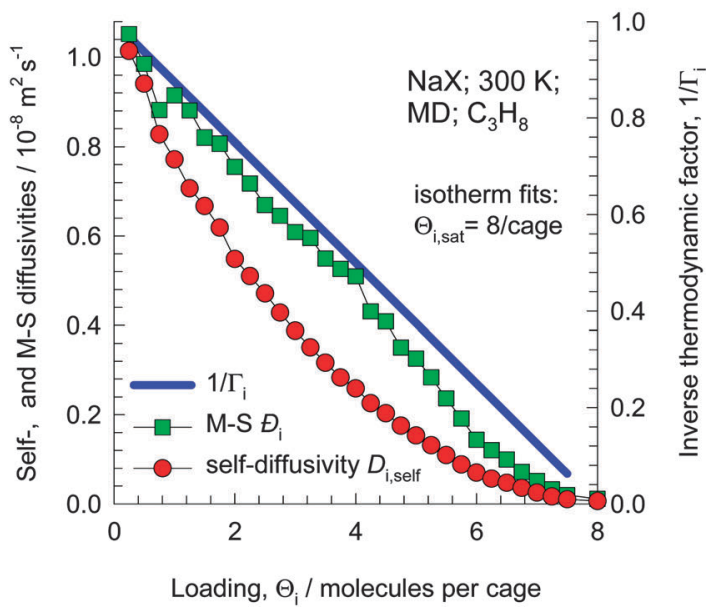

(c)
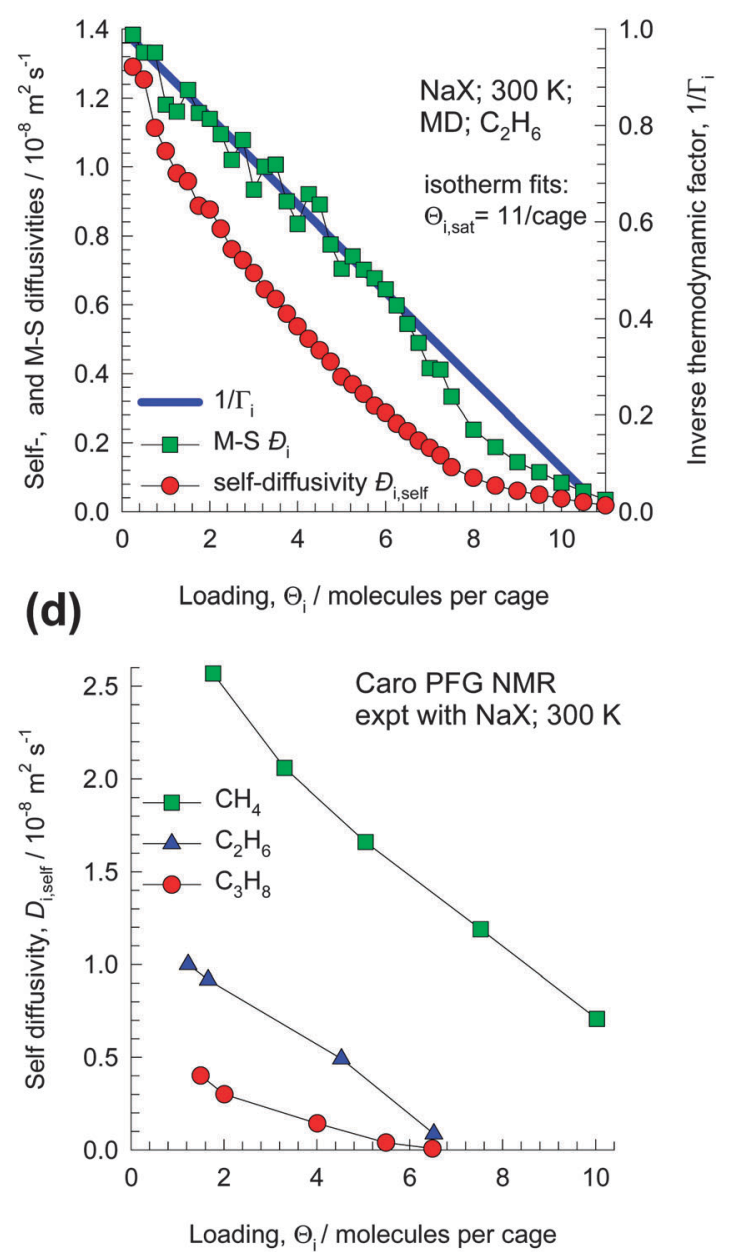

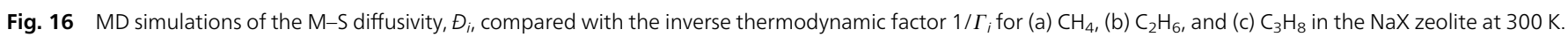
(d) PFG NMR experiments for the loading dependence of the self-diffusivities of $\mathrm{CH}_{4}, \mathrm{C}_{2} \mathrm{H}_{6}$, and $\mathrm{C}_{3} \mathrm{H}_{8}$ in the NaX zeolite. ${ }^{45}$

$\mathrm{CO}_{2}$ molecules to undergo a rotational motion when hopping from one $\mathrm{Mg}$ atom to a neighbouring one. Such rotational motion is observed in video animations of MD simulations, ${ }^{64}$ and has also been established experimentally. ${ }^{65}$ The attachment of alkynes and alkenes to the Fe atoms of FeMOF-74 is side-on, as determined by neutron diffraction; rotational motion is not to be expected in this scenario. Bao et al. ${ }^{4,66}$ report measurements of the transient uptake of $\mathrm{CO}_{2}, \mathrm{CH}_{4}$, $\mathrm{C}_{2} \mathrm{H}_{4}, \mathrm{C}_{2} \mathrm{H}_{6}, \mathrm{C}_{3} \mathrm{H}_{6}$, and $\mathrm{C}_{3} \mathrm{H}_{8}$ in MgMOF-74 crystals. The fitted $D_{i} / r_{\mathrm{c}}{ }^{2}$ values for $\mathrm{CO}_{2}, \mathrm{CH}_{4}, \mathrm{C}_{2} \mathrm{H}_{4}, \mathrm{C}_{2} \mathrm{H}_{6}, \mathrm{C}_{3} \mathrm{H}_{6}$, and $\mathrm{C}_{3} \mathrm{H}_{8}$ are $0.0075,0.045,0.006,0.012,0.00203$, and $0.00375 \mathrm{~s}^{-1}$, respectively. The diffusivity of $\mathrm{CO}_{2}$ is about a factor of 5 lower than that of $\mathrm{CH}_{4}$; this lower value cannot be rationalized easily on the basis of differences in molar masses or kinetic diameters. The lower diffusivity of $\mathrm{CO}_{2}$ is primarily the consequence of the significantly higher binding energy ( $c f$. Fig. $5 \mathrm{~b}$ ). Fig. 14b presents a plot of $\Phi_{i} / r_{\mathrm{c}}^{2}$ as a function of $-Q_{\mathrm{st}}$. It is remarkable to note that the diffusivity of the alkene is a factor of 2 lower than that of the corresponding alkane; this trend is opposite to that suggested by both molar masses and kinetic diameters.
The NiMOF-74 membrane permeation data of Lee et al. ${ }^{67}$ have been re-interpreted ${ }^{64}$ to obtain the values of the membrane transport coefficient, $\rho \mathrm{D}_{i} / \delta$, of $\mathrm{H}_{2}, \mathrm{~N}_{2}, \mathrm{CH}_{4}, \mathrm{CO}_{2}$ in NiMOF-74 as a function of $-Q_{\mathrm{st}}$; see Fig. 14c. We note that the transport coefficient of $\mathrm{CO}_{2}$ is more than an order of magnitude lower than that of $\mathrm{CH}_{4}$; this lowering is explainable on the basis of the trends in the isosteric heats of adsorption.

The results presented in Fig. 14 have implications for separations of $\mathrm{CO}_{2}-\mathrm{CH}_{4}$ and alkene-alkane mixtures using MgMOF-74 and NiMOF-74 membranes. ${ }^{5,17}$ While the stronger binding of $\mathrm{CO}_{2}$ and alkenes enhances the adsorption selectivity, there is a corresponding reduction in the diffusion selectivity. ${ }^{17}$

Further evidence of the strong influence of binding energy on diffusivities is provided by the data for $\nexists_{i}$ for $\mathrm{CO}_{2}$ in FAU-Si (all-silica), $\mathrm{NaY}\left(54 \mathrm{Na}^{+}\right.$per unit cell), and $\mathrm{NaX}\left(86 \mathrm{Na}^{+}\right.$per unit cell, pictured in Fig. 15a). The strong decrease, by about one order of magnitude, in the $\mathrm{CO}_{2}$ diffusivity with addition of $\mathrm{Na}^{+}$ cations, evidenced in the data for $\mathrm{M}-\mathrm{S}$ diffusivities ( $c f$. Fig. 15b), is ascribable to the much stronger binding of $\mathrm{CO}_{2}$ with the cations. Taking the isosteric heats of adsorption, $-Q_{\text {st }}$, to be a 
measure of the binding energy, we find a good correlation between $\bigoplus_{i}(0)$ and $-Q_{\text {st }}$; see Fig. 15c. The results presented in Fig. 15 have ramifications for separations of $\mathrm{CO}_{2}$-bearing mixtures. Increasing the number of cations increases the adsorption selectivity with respect to $\mathrm{CO}_{2}$, but this increase is achieved at the cost of diffusion selectivity which becomes increasingly in favor of its partner molecule. ${ }^{17}$

\section{Influence of $1 / \Gamma_{i}$ on the loading dependence of diffusivities}

We now focus on the quantification of the concentration dependence of $\bigoplus_{i}$ in microporous structures with characteristic channel dimensions larger than say $5 \AA$ A. A molecule can jump from one adsorption site to an adjacent one, provided it is not already occupied. The simplest model to describe the concentration dependence of the $\mathrm{M}-\mathrm{S}$ diffusivity is to assume that the frequency of molecular jumps is proportional to the fraction of total sites that is available for occupation.

$$
\bigoplus_{i}=\bigoplus_{i}(0) \frac{1}{\Gamma_{i}}
$$

where, in view of eqn (9), we consider $1 / \Gamma_{i}$ to be a generalized representation of the fractional vacancy.

Eqn (16) is a reasonable approximation for concentration dependence of the M-S diffusivity of $\mathrm{CH}_{4}, \mathrm{C}_{2} \mathrm{H}_{6}$, and $\mathrm{C}_{3} \mathrm{H}_{8}$ in the $\mathrm{NaX}$ zeolite, as demonstrated by comparisons of the $\mathrm{MD}$ data with the corresponding data for the inverse thermodynamic factor $1 / \Gamma_{i}$, obtained by differentiation of the CBMC simulated isotherm fits in Fig. 16. For all three guest molecules, $\bigoplus_{i}$ is nearly proportional to $1 / \Gamma_{i}$. Also plotted in Fig. $16 \mathrm{a}-\mathrm{c}$ are the MD data for the self-diffusivities, $D_{i, \text { self }}$, which show a more complex loading dependence because they additionally include correlation effects. PFG NMR experiments of Caro et al. ${ }^{45}$ for the loading dependence of the self-diffusivities of $\mathrm{CH}_{4}, \mathrm{C}_{2} \mathrm{H}_{6}$, and $\mathrm{C}_{3} \mathrm{H}_{8}$ in the NaX zeolite ( $c f$. Fig. $16 \mathrm{~d}$ ) confirm the validity of the MD simulation data. MD data similar to those presented in Fig. 16 hold for a number of other guest-host combinations; these data are provided in the ESI $\dagger$ accompanying this article. More detailed investigations ${ }^{43,44,68}$ of the loading dependencies of $\mathrm{CH}_{4}$ diffusivities reveal that subtle deviation from eqn (16) occurs for channel structures such as MFI, BEA, BOG, and ISV.

Preferential location of molecules within the structural framework can cause strong isotherm inflections. For example, (a)

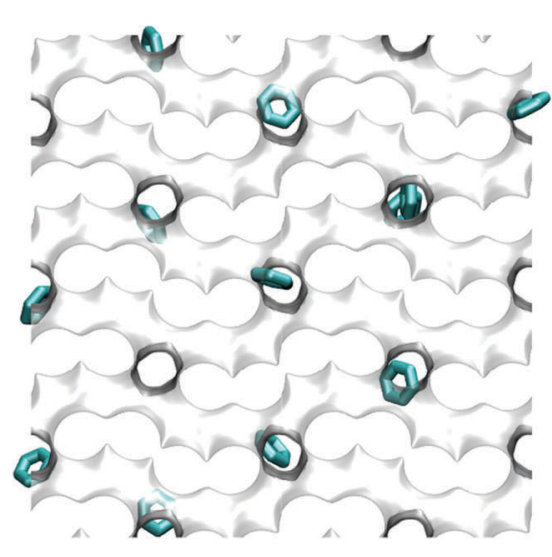

(b)

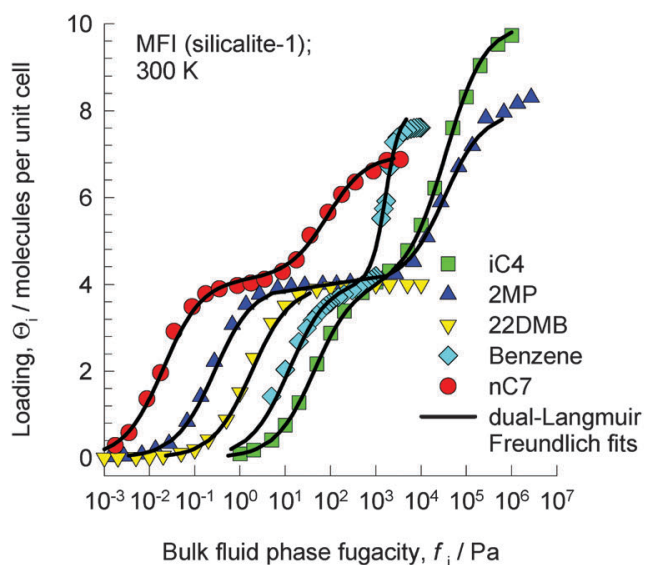

(c)

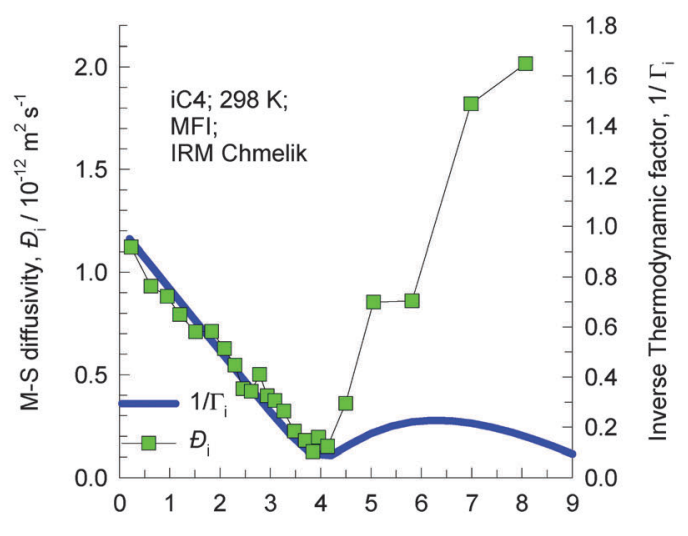

(d)

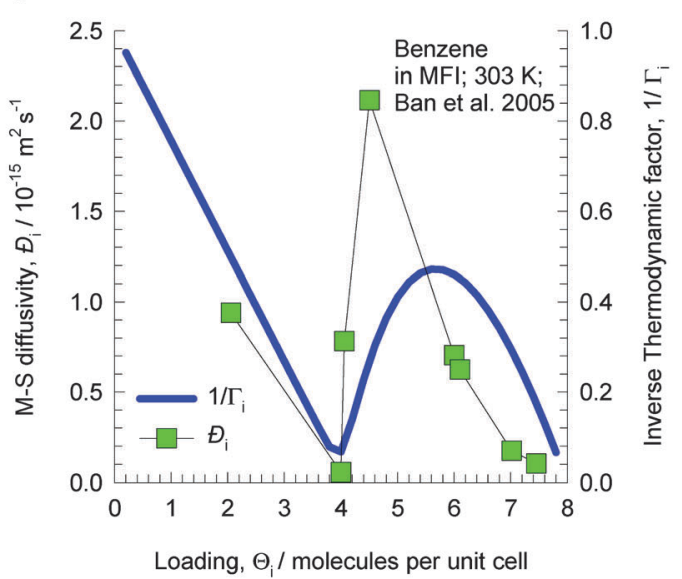

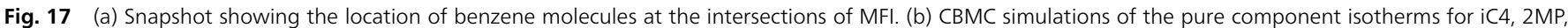

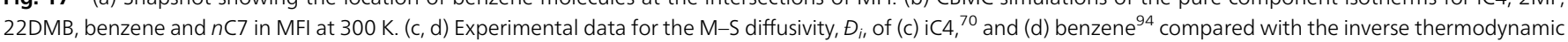
factor $1 / \Gamma_{i}$. Note that the data of Ban et al. ${ }^{94}$ for benzene were reported for the Fick diffusivity; these data were corrected to obtain the $\mathrm{M}-\mathrm{S} \Xi_{i}$. 
(a)

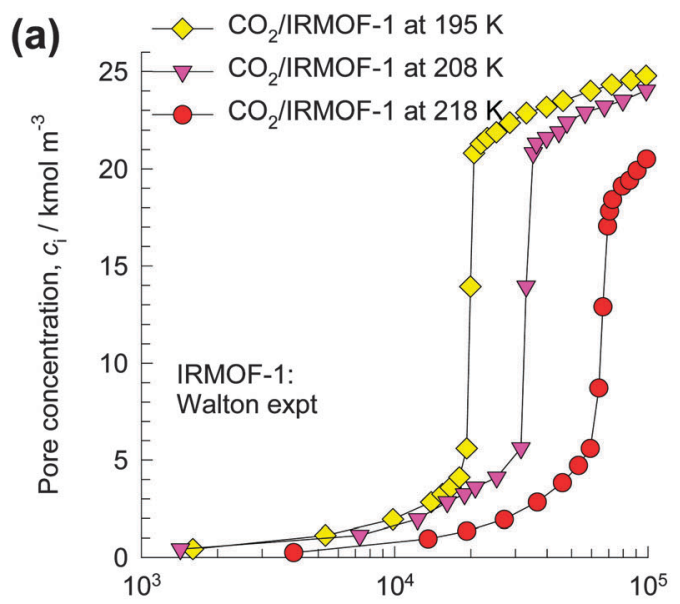

Bulk fluid phase pressure, $p_{\mathrm{i}} / \mathrm{Pa}$

(b)

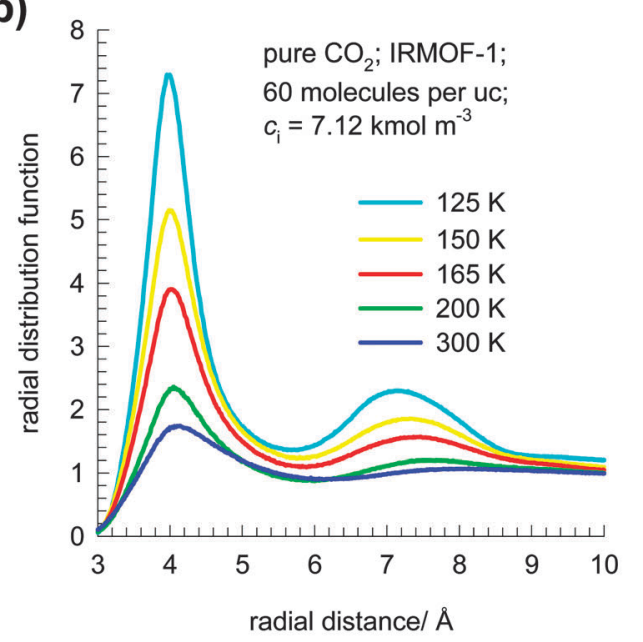

(c)

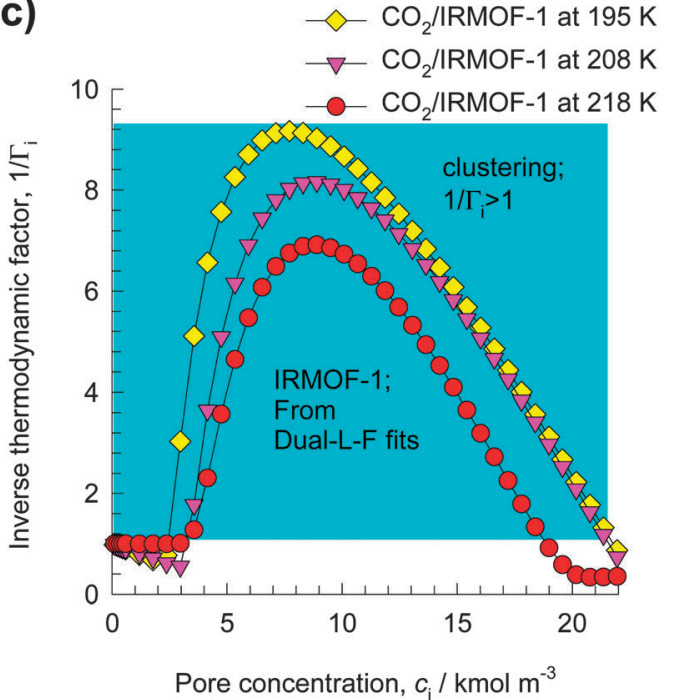

Fig. 19 (a) Experimental data ${ }^{96}$ for $\mathrm{CO}_{2} / \mathrm{IRMOF}-1$ isotherms at different temperatures, $T$. (b) Radial distribution function (RDF) for adsorption of $\mathrm{CO}_{2}$ in IRMOF-1 at various $T$ for a loading of 60 molecules per unit cell corresponding to $7.12 \mathrm{kmol} \mathrm{m}^{-3}$. (c) The inverse thermodynamic factor, $1 / \Gamma_{i}$, for $\mathrm{CO}_{2} /$ IRMOF-1 at different $T$, obtained from analytic differentiation of the dual-LangmuirFreundlich fits. 
unit cell of MFI. This implies that to obtain loadings higher than $\Theta_{i}=4$ molecules per unit cell, an extra "push" will be required to locate the molecules elsewhere within the channels; this leads to isotherm inflection; see isotherms of iso-butane (iC4), benzene, 2-methylpentane (2MP), and 2,2dimethylbutane (22DMB) in Fig. 17b. Due to strong isotherm inflections, the $1 / \Gamma_{i}$ exhibits a cusp-like inflection at a loading of $\Theta_{i}=4$, when all the preferred adsorption sites are occupied, as demonstrated for iC4 and benzene in Fig. 17c and d. In the range $0<\Theta_{i}<4,1 / \Gamma_{i}$ decreases nearly linearly with $\Theta_{i}$ signifying the fact that the vacancy decreases almost linearly with loading. For $\Theta_{i}>4,1 / \Gamma_{i}$ increases with $\Theta_{i}$ because additional sites within the MFI channels are created to accommodate more than 4 molecules per unit cell, i.e. the number of available sites increases within this loading range. These additional sites are within the channels, requiring the additional "push" that caused the inflection. On the basis of eqn (16) we should expect inflections in $1 / \Gamma_{i}$ vs. $\Theta_{i}$ to result in a corresponding cusp-like inflection in the $D_{i} v s$. $\Theta_{i}$ dependence. This is verified by the experimental data of Chmelik et al. ${ }^{70}$ ( $c f$. Fig. 17c), determined from transient uptake of iC4 within MFI crystals using infra-red microscopy (IRM). For loadings $\Theta_{i}>4$, the predictions of eqn (16) are not in quantitative agreement with the experimental data; the deviations are caused due to adsorbateadsorbate interactions that become increasingly significant at $\Theta_{i}>4$ due to proximity of iC4 molecules that are located close to one another along the channels. ${ }^{70}$

Benzene molecules locate preferentially at the intersections of MFI, where they also have an additional rotational degree of freedom; video animations included as ESI $\dagger$ demonstrate rotations. Experimental data for diffusivity of benzene in MFI also exhibit a similar cusp-like dependence on loading ( $c f$. Fig. 17d) with a minimum at $\Theta_{i}=4$ at which all intersection sites are filled up. With increase in loading $\Theta_{i}>4$ there is a loss of the rotational degree of freedom; concomitantly there is a sharp increase in the diffusivity. The entropic loss contributes directly to enhanced mobility. The loading dependence of the diffusivity also follows the trend dictated by $1 / \Gamma_{i}$.

Isotherm inflection is also observed for adsorption of $n$-heptane ( $n \mathrm{C} 7)$ in MFI ( $c f$. Fig. 17b). In this case the inflection is caused because the length of an $n \mathrm{C} 7$ molecule is slightly longer than the distance between intersections $;^{71}$ see the snapshot in Fig. 18a. The $1 / \Gamma_{i}$ vs. $\Theta_{i}$ curve for $n \mathrm{C} 7$ also exhibits a cusp-like inflection, which leaves its imprint on the loading dependence; see Fig. 18b. Similar inflection characteristics have been shown to manifest for $n \mathrm{C} 8$ and $n \mathrm{C} 9$ diffusion in MFI. ${ }^{72}$ Normal hexane ( $n$ C6) exhibits only a slight inflection at $\Theta_{i}=4$, because the length of the $n$ C6 molecule is practically commensurate with the distance between intersections, and the inflection characteristics of $\mathbf{M}-\mathbf{S}$ diffusivity are far less pronounced; see Fig. 18c.

The preferential location of branched and cyclic hydrocarbons at the channel intersections of the MFI zeolite has implications for the diffusivity of partner molecules in binary mixtures. In the PFG NMR investigation of Fernandez et al. ${ }^{73}$ the self-diffusivity of $n$-butane $(n \mathrm{C} 4)$ in mixtures with iso-butane
(iC4) was found to decrease by about two orders of magnitude as the loading of iC4 is increased from $\Theta_{\mathrm{iC} 4}=0$ to 2 molecules per unit cell. The reason for this strong decline can be understood on the basis of the preferential location of iC4 at the channel intersections of MFI. For $\Theta_{\mathrm{iC} 4}=2$, half the total number of intersections are occupied by iC4, that has a diffusivity which is about three orders of magnitude lower than that of $n \mathrm{C} 4$. Since the occupancy of the intersections is distributed randomly, each of the straight channels has an iC4 molecule ensconced somewhere along the channels; this is tantamount to blockage and leads to severe reduction in the molecular traffic of the intrinsically more mobile $n \mathrm{C} 4$. PFG NMR studies of Förste $e t a l^{74}$ found that the self-diffusivity of $\mathrm{CH}_{4}$ in MFI is significantly reduced as the loading of the co-adsorbed benzene increases. The explanation is again to be found in the hindering of $\mathrm{CH}_{4}$ diffusion due to blocking of the intersections by benzene. ${ }^{74}$ Koriabkina et $a l^{75}$ have reported experimental data for the self-diffusivities of both $n$ C6 and 2MP in C6-2MP mixtures. Their data, which were measured at $433 \mathrm{~K}$, show that both self-diffusivities are significantly reduced with increasing loading of $2 \mathrm{MP}$ in the mixture.
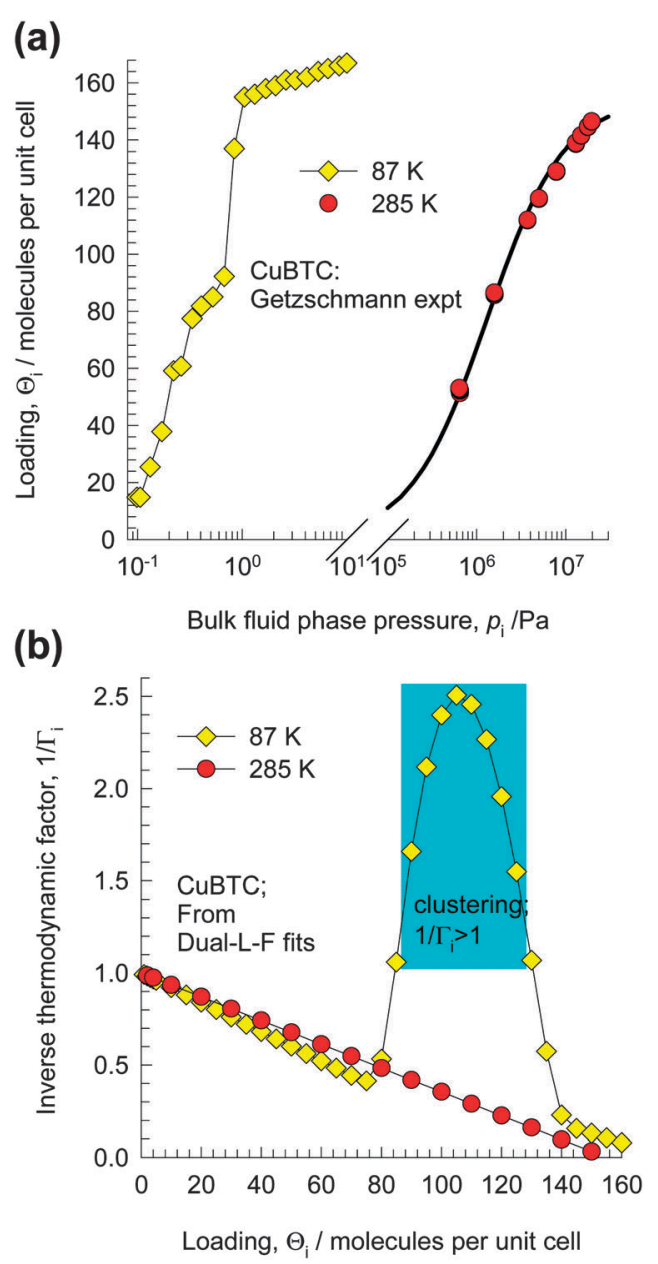

Fig. 20 (a) Experimental data ${ }^{79}$ for $\mathrm{CH}_{4}-\mathrm{CuBTC}$ isotherms at $T=87 \mathrm{~K}$, and $285 \mathrm{~K}$. (b) The inverse thermodynamic factor, $1 / \Gamma_{i}$ for $\mathrm{CH}_{4}-\mathrm{CuBTC}$ at different $T$, obtained from analytic differentiation of the dual-Langmuir-Freundlich fits. 


\section{Influence of $1 / \Gamma_{i}$ on systems exhibiting molecular clustering}

When the temperature $T$ is lower than the critical temperature, $T_{\mathrm{c}}$, the adsorbed phase is in a meta-stable thermodynamic state, and this leads to the possibility of cluster formation. ${ }^{76,77}$ For example, the critical temperature of $\mathrm{CO}_{2}$ is $301 \mathrm{~K}$, and there is a significant amount of cluster formation for adsorption at temperatures lower than $301 \mathrm{~K}$. Due to cluster formation the isotherms become increasingly steeper as the temperature is lowered below $301 \mathrm{~K}$; this is witnessed by the experimental data for $\mathrm{CO}_{2}$ isotherms in IRMOF-1 at $195 \mathrm{~K}, 208 \mathrm{~K}$, and $218 \mathrm{~K}$; see Fig. 19a. The characteristic signature of cluster formation is that the adsorption isotherm exhibits a steep increase in the loading; the steepness increases at lower $T$. The increase in the degree of clustering as $T$ falls progressively below $T_{\mathrm{c}}$ is evident in the radial distribution functions in Fig. 19b. The first peak in the RDF occurs at an intermolecular distance of $4 \AA$, typical of dimers. The height of the first peak is significantly lowered as $T$ approaches $T_{\mathrm{c}}=301 \mathrm{~K}$. At $T=300 \mathrm{~K}$, the RDF of the first peak practically vanishes, suggesting a homogeneous cluster-free adsorbed phase. A molecular cluster can be regarded as a $k$-mer; for a dimer, $k=2$. A $k$-mer occupies the same vacant adsorbed site as a normal, unclustered molecule, and consequently the fractional vacancy can exceed unity. For the range of concentrations at which cluster formation occurs, we find $1 / \Gamma_{i}>1 .{ }^{78}$ As $T$ falls progressively below $T_{\mathrm{c}}, 1 / \Gamma_{i}$ exceeds unity to an increasingly greater extent indicating increased levels of clustering; see Fig. 19c.

For $\mathrm{CH}_{4}, T_{\mathrm{c}}=191 \mathrm{~K}$, and consequently the experimental isotherm for adsorption of $\mathrm{CH}_{4}$ in $\mathrm{Cu}_{3}(\mathrm{btc})_{2}(\mathrm{CuBTC}=$ HKUST-1) at $87 \mathrm{~K}$ exhibits steep characteristics and $1 / \Gamma_{i}>1$ in some concentration regions; see Fig. 20a and b. The neutron diffraction experiments of Getzschmann et al. ${ }^{79}$ provide conclusive evidence that the steepness of the isotherms is related to cluster formation.

Eqn (16) would lead us to expect that the sharp peak in the loading dependence of $1 / \Gamma_{i}$ would cause a corresponding peak in the loading dependence of $\Xi_{i}$. This expectation is fulfilled for the experimental data of Chmelik et al., ${ }^{80}$ obtained by transient uptake within crystals of CuBTC using Infrared Microscopy (IRM) of $n$-butane ( $\left.n \mathrm{C} 4, T_{\mathrm{c}}=425 \mathrm{~K}\right)$, iso-butane (iC4, $\left.T_{\mathrm{c}}=408 \mathrm{~K}\right)$, neopentane (neo-P, $T_{\mathrm{c}}=434 \mathrm{~K}$ ), and 2-methylbutane (2MB, $\left.T_{\mathrm{c}}=460 \mathrm{~K}\right)$ at $T=298 \mathrm{~K}$, significantly lower than the critical temperatures of each of the four guest species, see Fig. 21. The observed behaviours of $1 / \Gamma_{i}$ and $D_{i}$ for loadings $\Theta_{i}<8$ (a)

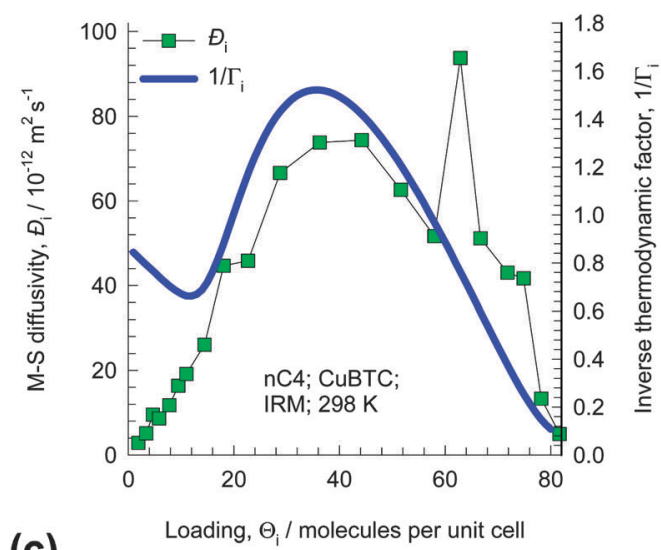

(c)

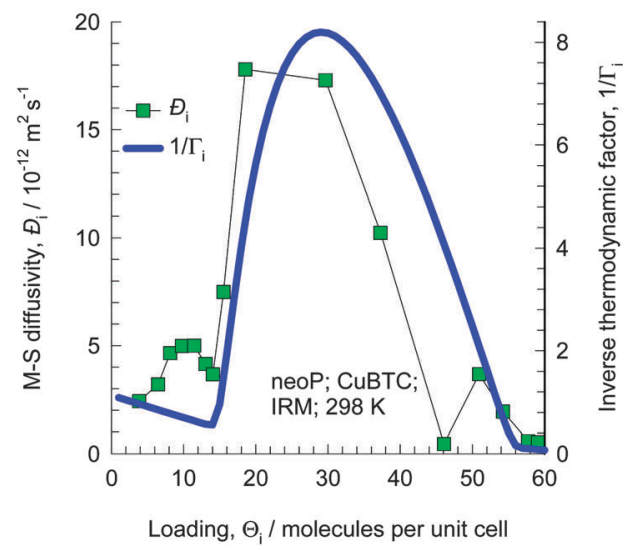

(b)

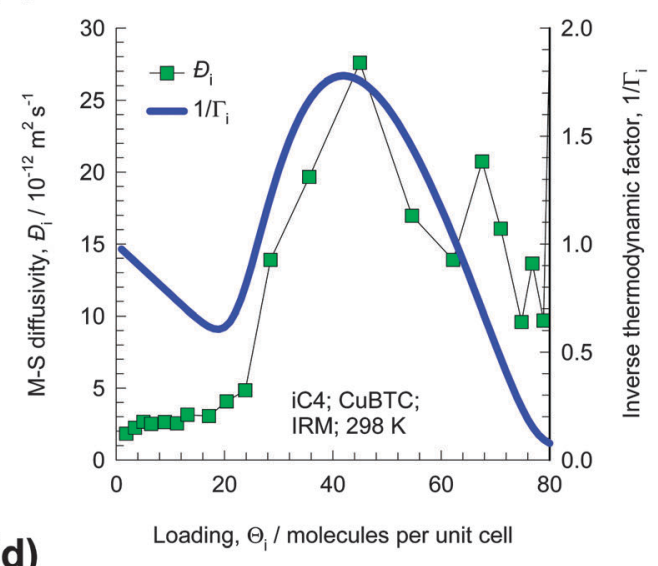

(d)

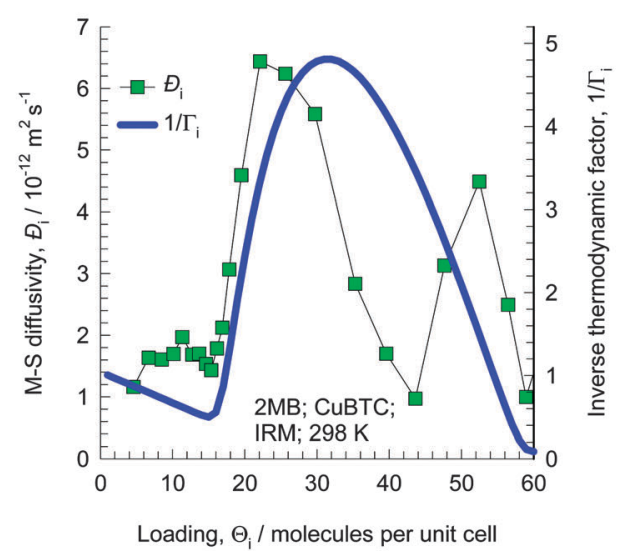

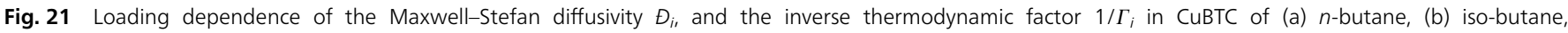

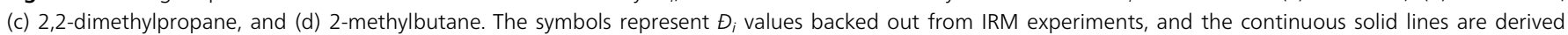
from IRM isotherm fits. These data are re-plotted using the information contained in Chmelik et al. ${ }^{80}$ 
(a)

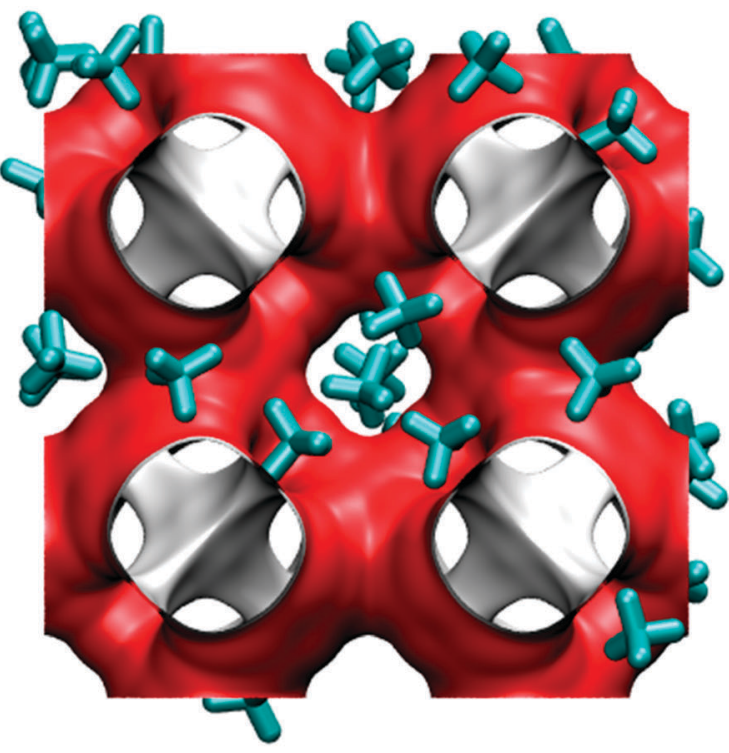

(b)

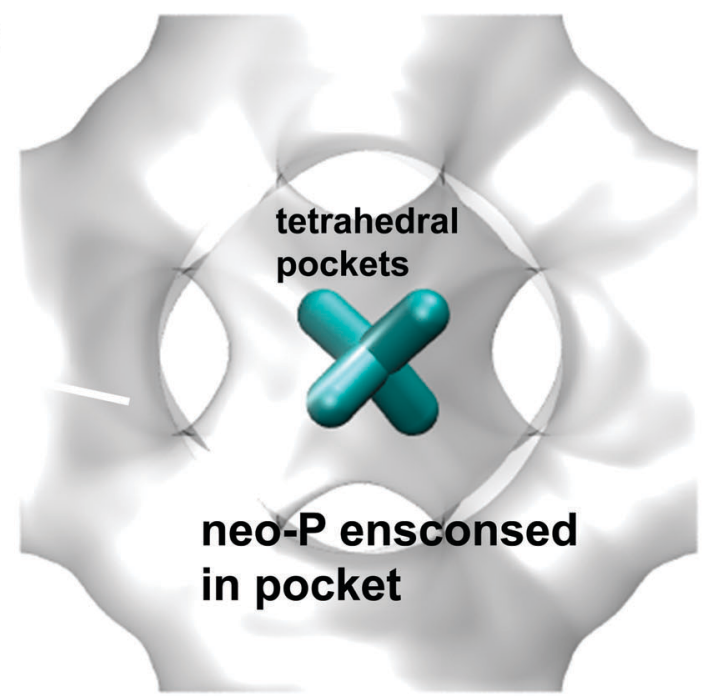

Fig. 22 (a) Pore landscape of CuBTC showing the adsorbed neopentane (neo-P) molecules. (b) Snapshot showing the location of neo-pentane lodged within a tetrahedral pocket.

molecules per unit cell need special attention and explanation. The CuBTC structure consists of two types of "cages" and two types of "windows" separating these cages. Large cages are inter-connected by $9 \AA$ windows of square cross-section. The large cages are also connected to tetrahedral-shaped pockets of ca. 6 Å size through triangular-shaped windows of $c a .4 .6 \AA$ size. There are 8 tetrahedral pockets per unit cell, and these are preferred locations of molecules at low loadings. Each pocket can accommodate only one of $n \mathrm{C} 4$, iC4, neo-P or $2 \mathrm{MB}$; this is illustrated by the snapshot in Fig. 22 for neo-P adsorbed in CuBTC. For loadings $\Theta_{i}<8$ molecules per unit cell, the alkanes prefer location in the pockets, and the diffusion characteristics are dictated by hops across triangular windows of $4.6 \AA$. Consequently the diffusivities tend to be low. The diffusivities of molecules inhabiting the larger cages are about an order of magnitude higher because they correspond to hops across larger $9 \AA$ windows. As the molecules begin to populate the larger cages, the $D_{i}$ increases sharply till a maximum is reached.

The strong confinement, and binding, of the $n \mathrm{C} 4$, iC4, neo-P and $2 \mathrm{MB}$ molecules within the tetrahedral pockets of CuBTC also gets reflected in much higher values of the isosteric heats of adsorption, $-Q_{\text {st }}$. This is demonstrated in the plots comparing the dependencies of $\theta_{i}$ and the corresponding $-Q_{\mathrm{st}}$, determined from molecular simulations; see Fig. 23. For loadings $\Theta_{i}<8$ molecules per unit cell, the low diffusivity values of $D_{i}$ are caused by the stronger binding and confinement within the pockets. For loadings $\Theta_{i}>8$ molecules per unit cell, the loading is much more strongly dependent on the characteristics of $1 / \Gamma_{i}$.

The experimental investigations of Salles et al. ${ }^{81,82}$ for $\mathrm{CO}_{2}$ diffusion in MIL-47 and MIL-53(Cr)-lp provide further confirmation of the unusual trends resulting from clustering at $T=230 \mathrm{~K}<T_{\mathrm{c}}$. Molecular clustering is a plausible explanation for the maximum in the PFG NMR data ${ }^{83}$ for the loading dependence of $n$ C6 diffusivity in Na75CaX and Na30CaX at $T=293 \mathrm{~K}$, significantly lower than $T_{\mathrm{c}}=507 \mathrm{~K}$; see Fig. 24a. Heink et al..$^{83}$ offered an alternative explanation for the observed maximum in the loading dependence of $n \mathrm{C} 6$; this is based on the relative magnitudes of adsorbate-adsorbate and adsorbatecation interactions. The authors argue that the relative magnitudes of these two interactions are altered with increasing loadings, causing a maximum to occur.

The observed maxima in the loading dependencies of the PFG NMR data ${ }^{84}$ on self-diffusivities of benzene $\left(T_{\mathrm{c}}=562 \mathrm{~K}\right)$, toluene $\left(T_{\mathrm{c}}=592 \mathrm{~K}\right)$, and $p$-xylene $\left(T_{\mathrm{c}}=616 \mathrm{~K}\right)$ in the NaX zeolite at $393 \mathrm{~K}$ ( $c f$. Fig. 24b) are also ascribable to molecular clustering that manifest at temperatures significantly lower than the critical temperature. A similar maximum in the loading dependence has been reported for diffusivity of methanol in the NaX zeolite at $373 \mathrm{~K}^{85}$ see Fig. $24 \mathrm{c}$. To demonstrate that the cause of molecular clustering is the hydrogen bonding between molecular pairs, Fig. 25a presents data for the radial distribution functions (RDFs) for distances between all combinations of $\mathrm{O}$ and $\mathrm{H}$ atoms of pairs of methanol and ethanol molecules adsorbed within the pores of FAU-Si. We note that the first peaks in the RDFs occur at an intermolecular distance of $<2 \AA$, which is characteristic of hydrogen bonding. ${ }^{86}$ Molecular simulation data for the loading dependence of $D_{i}$ and $1 / \Gamma_{i}$ for methanol and ethanol are shown in Fig. 25b and c. Both diffusivity and the inverse thermodynamic factor exhibit a maximum in their loading dependencies, and the behaviour is similar to that observed for methanol diffusivity in NaX, as shown in Fig. 24c.

The loading dependencies portrayed in Fig. 24 and 25 conform with Type IV in the classification of Kärger and Pfeifer ${ }^{87}$ and our analysis here establishes that the curious maximum in each case is attributable to molecular clustering. ${ }^{78}$

A further consequence of molecular clustering is that in concentration regions where $1 / \Gamma_{i}>1$, eqn (4) and (6) would yield the hierarchy $D_{i}<D_{i} \leq D_{i, \text { self. }}$. Experimental confirmation 
(a)

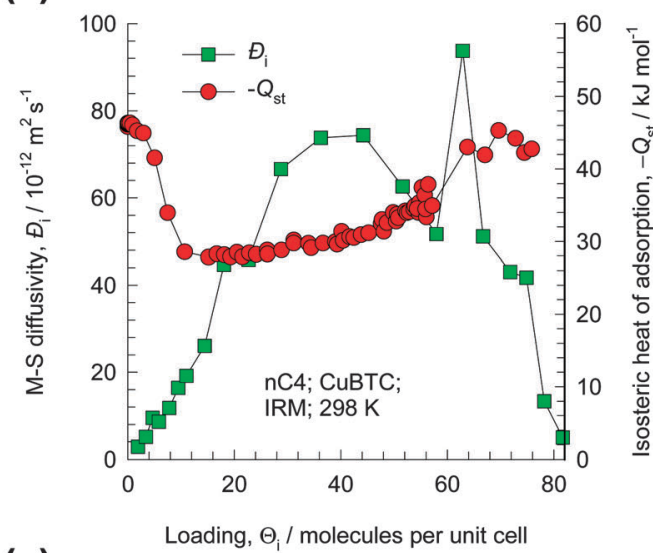

(c)

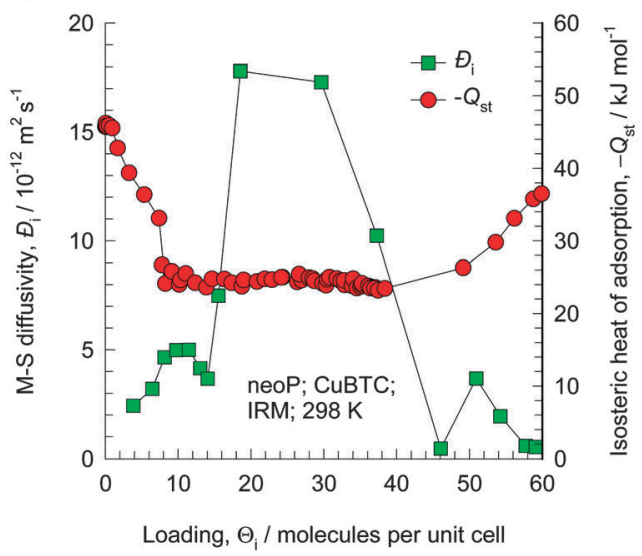

(b)

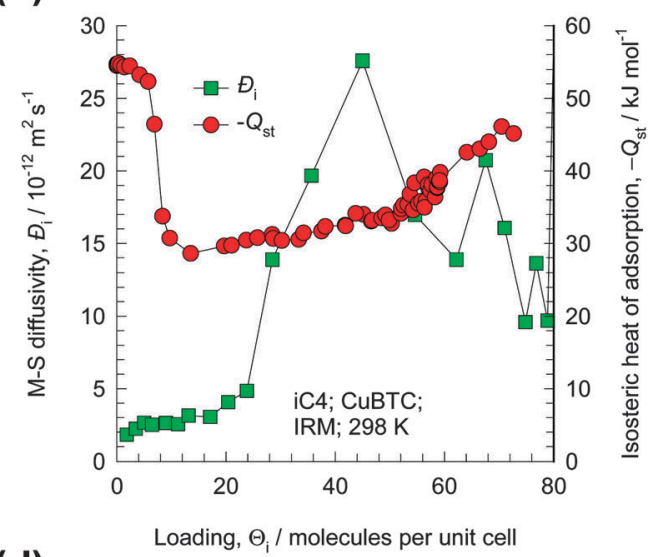

(d)

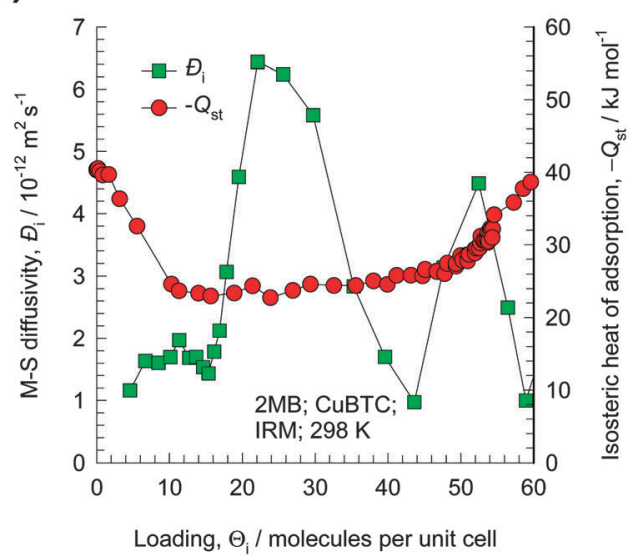

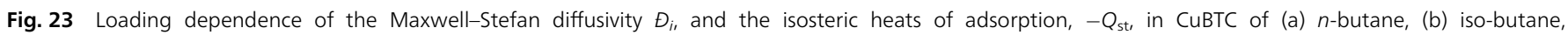

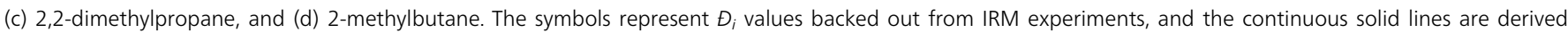
from IRM isotherm fits. These data are re-plotted using the information contained in Chmelik et al. ${ }^{80}$

of this unusual hierarchy is found for diffusion of methanol and ethanol in ZIF-8. ${ }^{88}$

Tsotsalas et al. ${ }^{89}$ have investigated the kinetics of desorption of 1-butanol, diethyl ether, and $n$-pentane in CuBTC and found that molecular clustering effects, along with $1 / \Gamma_{i}>1$, provide an explanation for unusual desorption behaviours.

\section{Influence of thermodynamic correction factors on mixture diffusion}

Mixture diffusion is influenced by thermodynamic coupling, that is quantified by the matrix of thermodynamic correction factors, $\Gamma_{i j}$

$$
\Gamma_{i j}=\frac{q_{i}}{f_{i}} \frac{\partial f_{i}}{\partial q_{j}} ; i, j=1, \ldots, n
$$

The non-diagonal elements of $\Gamma_{i j}$ cause thermodynamic coupling between species diffusion. ${ }^{17}$ Strong thermodynamic coupling can cause overshoots of component loadings during transient uptake inside crystals. Experimental data for transient uptake of $\mathrm{N}_{2}(1)-\mathrm{CH}_{4}(2)$ mixtures in crystallites of LTA-4A (cf. Fig. 26a), reported in the classic paper of Habgood, ${ }^{90}$ provide a good illustration of the influence of $\Gamma_{i j}$. The diffusivity of $\mathrm{N}_{2}$ is a factor of 22 higher than that of $\mathrm{CH}_{4}$, but it has an adsorption strength that is a factor of 2.2 lower. During the initial stages of the transient uptake, the pores of LTA-4A are predominantly richer in the more mobile $\mathrm{N}_{2}$, but this is displaced by the more strongly adsorbed, tardier $\mathrm{CH}_{4}$ molecules at longer times resulting in a curious maximum in the $\mathrm{N}_{2}$ uptake. The continuous solid lines in Fig. 26a are calculations of the Maxwell-Stefan mixture diffusion model ${ }^{47}$ that takes proper account of thermodynamic coupling. The $\mathrm{N}_{2}$ overshoot is adequately captured by the $\mathrm{M}-\mathrm{S}$ model that assumes both diffusivities $\bigoplus_{i}$ to be loading independent. The dashed lines are calculations in which thermodynamic coupling effects are ignored, and the elements of $\Gamma_{i j}$ are assumed to be the Kronecker delta, $\delta_{i j}$; in this case no overshoot is observed. The $\mathrm{N}_{2}$ overshoot is purely a result of thermodynamic coupling quantified by finite off-diagonal elements of $\Gamma_{i j}$.

The experimental data of Kärger and Bülow ${ }^{91}$ for transient uptake of the $n$-heptane(1)-benzene(2) mixture in the NaX zeolite at $359 \mathrm{~K}$ show an overshoot in the uptake of the more mobile $n$-heptane, see Fig. 26b. Benzene is much more strongly adsorbed due to electrostatic interactions with the $\mathrm{Na}^{+}$cations. The stronger binding energy of benzene makes the mobility of 
(a)

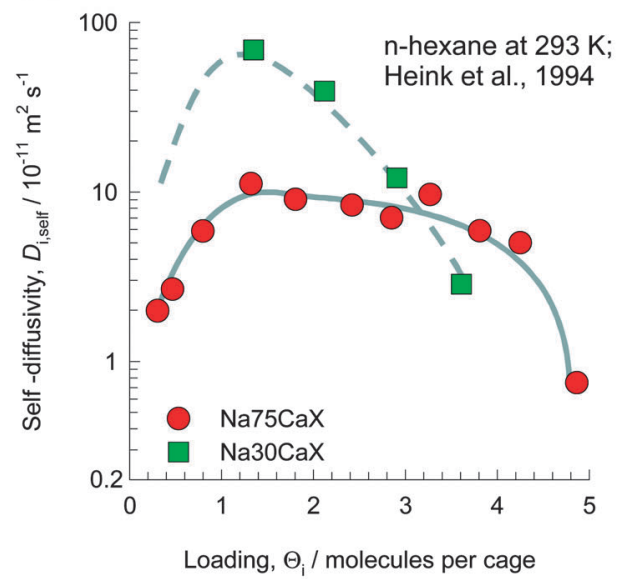

(b)

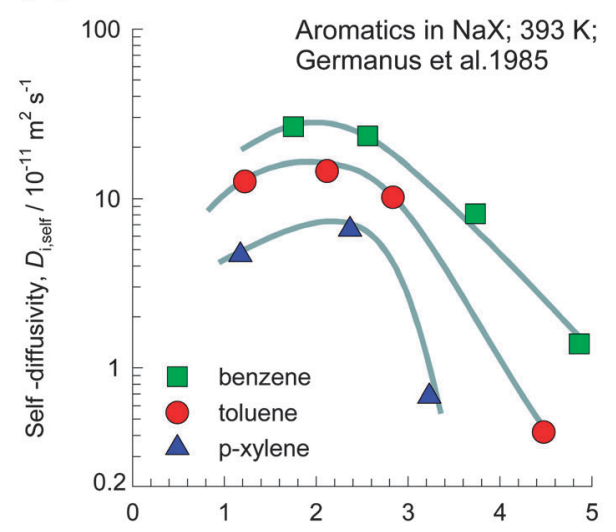

(c)

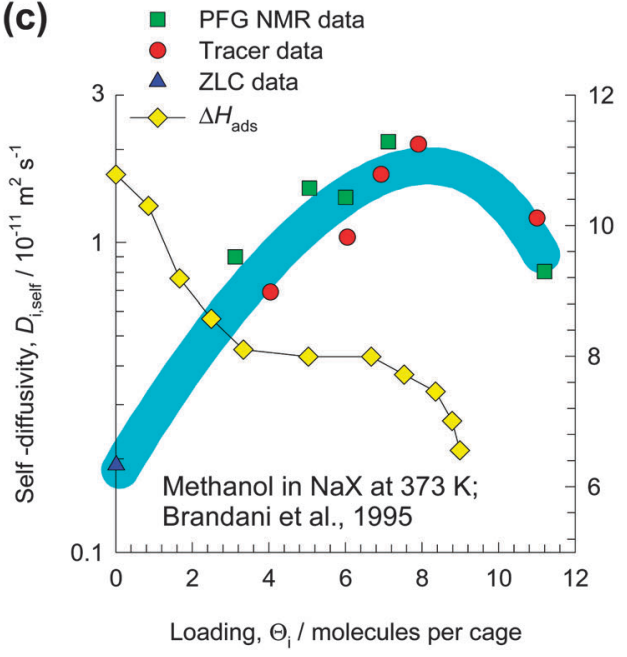

Fig. 24 (a) PFG NMR experimental data ${ }^{83}$ for the loading dependence of the self-diffusivity of $n$-hexane ( $n$ C6) in the NaCaX zeolite at $293 \mathrm{~K}$. (b) PFG NMR experimental data ${ }^{84}$ for the loading dependence of the self-diffusivity benzene, toluene and p-xylene in the NaX zeolite at $393 \mathrm{~K}$. (b) Experimental data for the loading dependence of the self-diffusivity of methanol in $\mathrm{NaX}^{85}$

benzene significantly lower than that of the non-polar $n$-heptane ${ }^{64}$ The overshoot in the $n$-heptane uptake is primarily due to thermodynamic coupling effects, quantified by finite offdiagonal elements of $\Gamma_{i j}$. The assumption of $\Gamma_{i j}=\delta_{i j}$ is not able to capture the overshoot of $n$-heptane. ${ }^{92}$ (a)
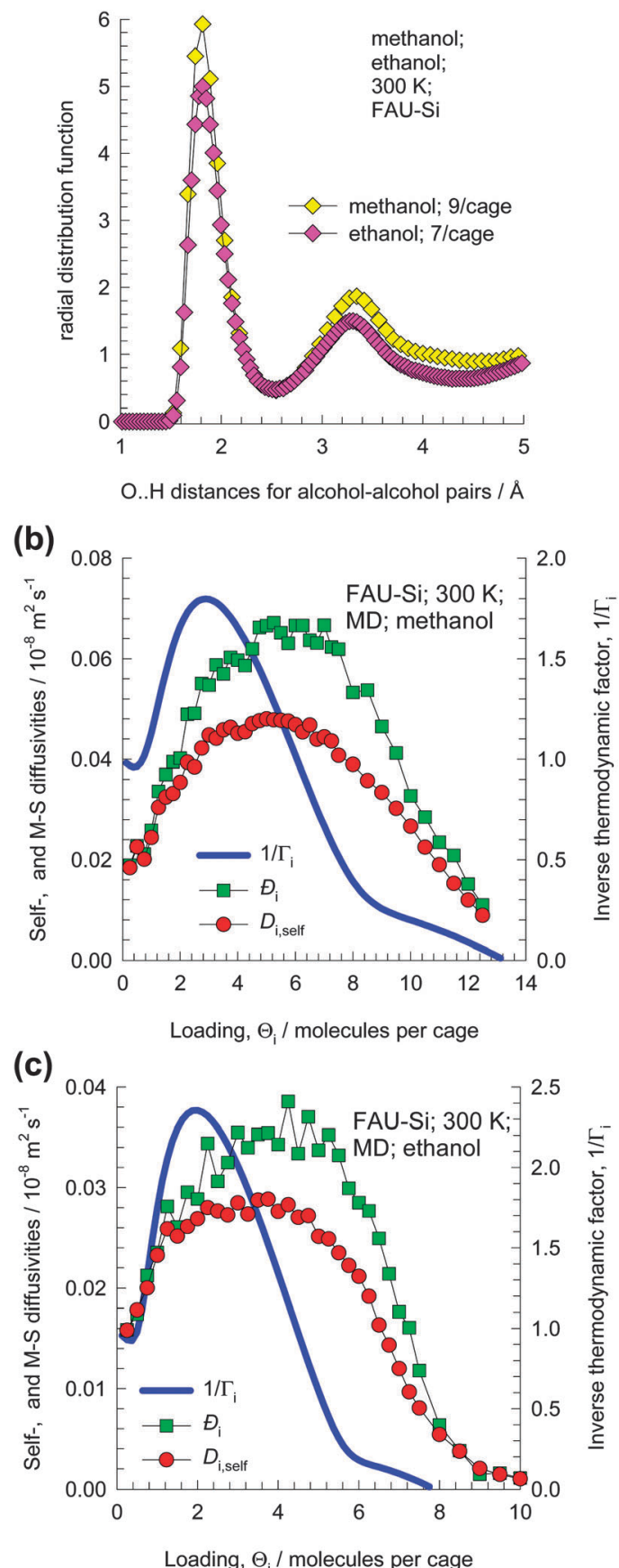

Fig. 25 (a) Radial distribution functions (RDFs) for methanol-methanol, and ethanol-ethanol molecular pairs in FAU-Si at $300 \mathrm{~K}$. The RDFs are constructed on the basis of distances between all the inter-molecular pairs of $\mathrm{O}$ and $\mathrm{H}$ atoms of molecular pairs. (b, c) Comparison of the loading dependencies of the $\mathrm{M}-\mathrm{S}$ diffusivity, $\Theta_{i}$, and $1 / \Gamma_{i}$ of (b) methanol, and (c) ethanol in FAU-Si at $300 \mathrm{~K}$; these data are obtained from molecular simulations. ${ }^{97}$

For transient uptake in CHA of mixtures of alcohols of different chain lengths, the alcohol with the smaller chain length will exhibit an overshoot due to thermodynamic coupling; simulation data are provided in the ESI. $\dagger$ 


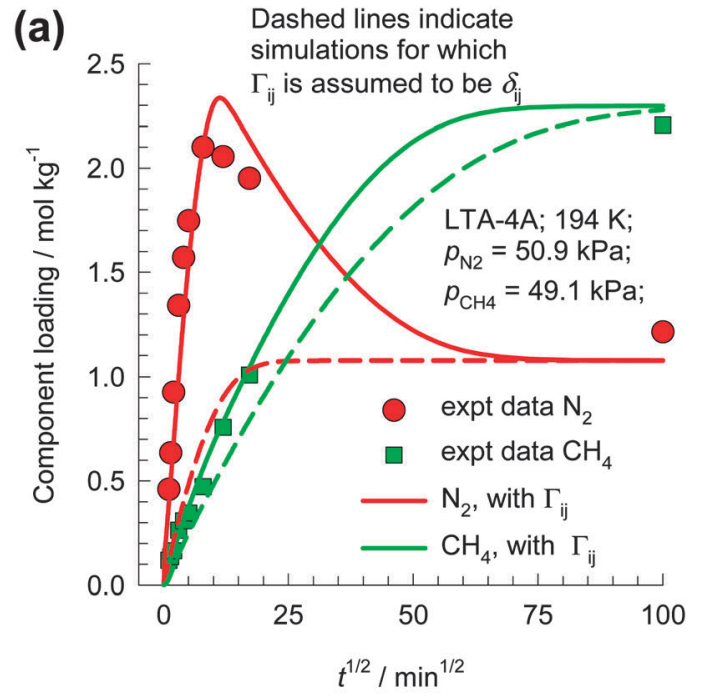

(b)

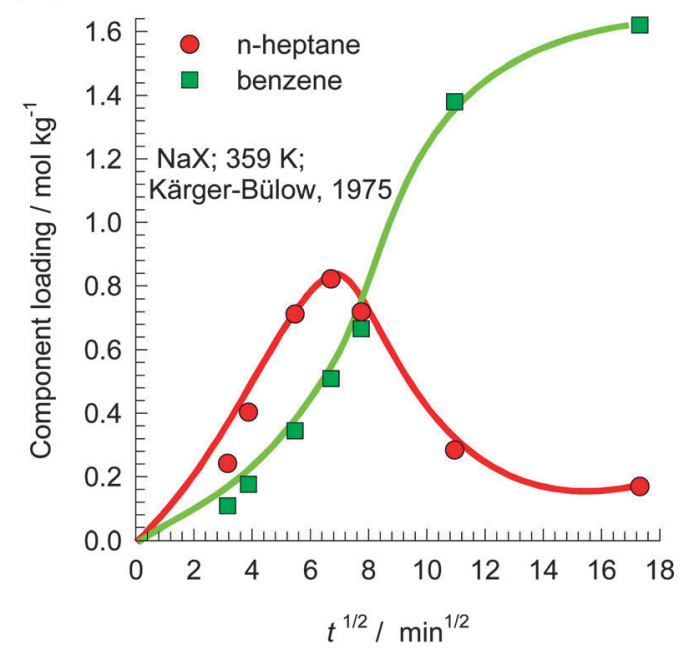

Fig. 26 (a) Experimental data of Habgood ${ }^{90}$ for transient uptake of $\mathrm{N}_{2}$ and $\mathrm{CH}_{4}$ within LTA-4A crystals, exposed to an equimolar binary gas mixture at $194 \mathrm{~K}$. The continuous solid lines are calculations of the Maxwell-Stefan model taking due account of thermodynamic coupling with non-diagonal elements $\Gamma_{i j}$. The dashed lines are calculations in which the thermodynamic coupling is ignored, and $\Gamma_{i j}$ is assumed to be the Kronecker delta, $\delta_{i j}$. (b) Experimental data of Kärger and Bülow ${ }^{91}$ for transient uptake of the $n$-heptane(1)-benzene(2) mixture in the NaX zeolite at $359 \mathrm{~K}$. Further information is provided in the ESI. $†$

\section{Conclusions}

The following major conclusions can be drawn regarding the influence of adsorption thermodynamics on the magnitude and loading dependence of diffusivities in ordered crystalline nanoporous materials.

(1) For cage-type structures in which adjacent cages are separated by narrow windows in the 3.2-4.4 $\AA$ size range, the loading dependence of the diffusivities is dictated by the free energy barriers at the window constrictions. Most commonly, the diffusivities increase in magnitude until a maximum is reached, and decrease thereafter till the adsorption saturation concentration, $c_{i \text {,sat }}$, is reached. Eqn (14), based on the quasi-chemical model to describe adsorbate-adsorbate interactions, provides a simple approach for describing the loading dependence. The parameter $\left(w_{i} / R T\right)$ can be estimated by fitting the isotherm. The zero-loading diffusivity $\bigoplus_{i}(0)$ is dictated primarily by the molecular size and the degree of constraint at the window regions.

(2) For diffusion in structures with characteristic channel dimensions larger than say $5 \AA$, there are often no discernible regions posing high free energy barriers for diffusion, and the loading dependence is primarily dictated by the inverse thermodynamic factor $1 / \Gamma_{i}$, following eqn (16). The zero-loading diffusivity $\bigoplus_{i}(0)$ is strongly influenced by the binding energy in this case.

(3) When no molecular clustering occurs, $1 / \Gamma_{i} \leq 1$, and we have the "normal" hierarchy of diffusivities: $D_{i \text {,self }} \leq \emptyset_{i} \leq D_{i}$.

(4) For temperatures significantly below the critical temperature, $T_{\mathrm{c}}$, molecular clustering may occur for a range of concentrations and this occurrence is signalled by $1 / \Gamma_{i}>1$ reflecting metastable equilibrium. The greater the extent of clustering of molecules, the larger is the value by which $1 / \Gamma_{i}$ exceeds unity. The extent of clustering increases as $T$ falls increasingly below $T_{\mathrm{c}}$. The degree of clustering is loading dependent, and approximately follows the trend of $1 / \Gamma_{i} v s . c_{i}$.

(5) For adsorption of water and alcohols, molecular clustering, induced by hydrogen bonding, causes the inverse thermodynamic factor $1 / \Gamma_{i}$ to exceed unity for a range of concentrations $c_{i}$. This has significant consequences on the loading dependence of the diffusivities.

(6) Non-monotonic variation of the diffusivity with increasing chain length of the linear molecule can occur due to match, or mis-match, between the molecular dimensions and the intrinsic periodicity of the framework.

The proper appreciation of the interplay between adsorption thermodynamics and diffusivities will result in improved models and procedures for development of separation technologies of the future.

\section{Nomenclature}

$b_{i} \quad$ dual-Langmuir-Freundlich adsorption constant for species $i, \mathrm{pa}^{-\nu_{i}}$

$c_{i}$ pore concentration of species $i, c_{i}=q_{i} / V_{\mathrm{p}}, \mathrm{mol} \mathrm{m}^{-3}$ $c_{i, \mathrm{sat}} \quad$ saturation pore concentration of species $i$, $c_{i, \text { sat }}=q_{i, \text { sat }} / V_{\mathrm{p}}, \mathrm{mol} \mathrm{m}^{-3}$

$d_{\mathrm{p}} \quad$ characteristic channel dimension, $\mathrm{m}$

$D_{i} \quad$ Fick diffusivity of species $i, \mathrm{~m}^{2} \mathrm{~s}^{-1}$

$D_{i, \text { self }} \quad$ self-diffusivity of species $i, \mathrm{~m}^{2} \mathrm{~s}^{-1}$

$Ð_{i} \quad$ Maxwell-Stefan diffusivity, $\mathrm{m}^{2} \mathrm{~s}^{-1}$

$\bigoplus_{i i} \quad$ self-exchange coefficient, $\mathrm{m}^{2} \mathrm{~s}^{-1}$

$\bigoplus_{i i, \mathrm{fl}}$

$\bigoplus_{i}(0)$

$\delta F_{i}$

$f_{i}$

$F$

$n_{i} \quad$ number of molecules of species $i$ in the simulation box, dimensionless 
$N_{i} \quad$ molar flux of species $i, \mathrm{~mol} \mathrm{~m}^{-2} \mathrm{~s}^{-1}$

$p_{i} \quad$ partial pressure of species $i, \mathrm{~Pa}$

$q_{i} \quad$ molar loading species $i, \mathrm{~mol} \mathrm{~kg}^{-1}$

$q_{i, \text { sat }} \quad$ saturation molar loading species $i, \mathrm{~mol} \mathrm{~kg}^{-1}$

$-Q_{\text {st }} \quad$ isosteric heat of adsorption, $\mathrm{J} \mathrm{mol}^{-1}$

$r_{\mathrm{c}} \quad$ radius of the crystallite, $\mathrm{m}$

$\mathbf{r}_{l, i}(t) \quad$ position vector for molecule $l$ of species $i$ at any time $t, \mathrm{~m}$

$R \quad$ gas constant, $8.314 \mathrm{~J} \mathrm{~mol}^{-1} \mathrm{~K}^{-1}$

$t \quad$ time, s

$T \quad$ temperature, $\mathrm{K}$

$T_{\mathrm{c}} \quad$ critical temperature, $\mathrm{K}$

$V_{\mathrm{p}} \quad$ accessible pore volume, $\mathrm{m}^{3} \mathrm{~kg}^{-1}$

$w_{\mathrm{i}} \quad$ nearest neighbour interaction energy in the lattice model, $\mathrm{J} \mathrm{mol}^{-1}$

$z \quad$ coordination number, dimensionless

Greek letters

$\delta \quad$ thickness of the membrane layer, $\mathrm{m}$

$\delta_{i j} \quad$ Kronecker delta, dimensionless

$\Gamma_{i} \quad$ thermodynamic factor, dimensionless

$\phi \quad$ fractional pore volume, dimensionless

$\nu_{i} \quad$ exponent in the dual-Langmuir-Freundlich isotherm, dimensionless

$\zeta_{i} \quad$ parameter defined by eqn (11), dimensionless

$\mu_{i} \quad$ molar chemical potential, $\mathrm{J} \mathrm{mol}^{-1}$

$\rho \quad$ framework density, $\mathrm{kg} \mathrm{m}^{-3}$

$\theta_{i} \quad$ fractional occupancy of species $i$, dimensionless

$\theta_{\mathrm{V}} \quad$ fractional vacancy of species $i$, dimensionless

$\Theta_{i} \quad$ loading of species $i$, molecules per unit cell or per cage

$\Theta_{i \text { sat }} \quad$ saturation loading of species $i$, molecules per unit cell or per cage

Subscripts

fl referring to the fluid phase

$i \quad$ referring to component $i$

sat referring to saturation conditions

$\mathrm{V} \quad$ referring to vacancy

\section{Notes and References}

1 J. Kärger, D. M. Ruthven and D. N. Theodorou, Diffusion in Nanoporous Materials, Wiley-VCH, Weinheim, 2012.

2 E. D. Bloch, L. Murray, W. L. Queen, S. M. Chavan, S. N. Maximoff, J. P. Bigi, R. Krishna, V. K. Peterson, F. Grandjean, G. J. Long, B. Smit, S. Bordiga, C. M. Brown and J. R. Long, J. Am. Chem. Soc., 2011, 133, 14814-14822.

3 Y.-S. Bae, C. Y. Lee, K. C. Kim, O. K. Farha, P. Nickias, J. T. Hupp, S. T. Nguyen and R. Q. Snurr, Angew. Chem., Int. Ed., 2012, 51, 1857-1860.

4 Z. Bao, S. Alnemrat, I. Vasiliev, Q. Ren, L. Yu, X. Lu and S. Deng, Langmuir, 2011, 27, 13554-13562.

5 E. D. Bloch, W. L. Queen, R. Krishna, J. M. Zadrozny, C. M. Brown and J. R. Long, Science, 2012, 335, 1606-1610.
6 Z. R. Herm, J. A. Swisher, B. Smit, R. Krishna and J. R. Long, J. Am. Chem. Soc., 2011, 133, 5664-5667.

7 M. C. Das, Q. Guo, Y. He, J. Kim, C. G. Zhao, K. Hong, S. Xiang, Z. Zhang, K. M. Thomas, R. Krishna and B. Chen, J. Am. Chem. Soc., 2012, 134, 8708-8710.

8 R. Krishna and J. M. van Baten, Phys. Chem. Chem. Phys., 2011, 13, 10593-10616.

9 Y. He, R. Krishna and B. Chen, Energy Environ. Sci., 2012, 5, 9107-9120.

10 S. C. Xiang, Y. He, Z. Zhang, H. Wu, W. Zhou, R. Krishna and B. Chen, Nat. Commun., 2012, 3, 954.

11 Y. He, W. Zhou, R. Krishna and B. Chen, Chem. Commun., 2012, 48, 11813-11831.

12 H. Wu, K. Yao, Y. Zhu, B. Li, Z. Shi, R. Krishna and J. Li, J. Phys. Chem. C, 2012, 116, 16609-16618.

13 D. Dubbeldam, R. Krishna, S. Calero and A. Ö. Yazaydın, Angew. Chem., Int. Ed., 2012, 51, 11867-11871.

14 J. A. Mason, K. Sumida, Z. R. Herm, R. Krishna and J. R. Long, Energy Environ. Sci., 2011, 4, 3030-3040.

15 W. Lu, J. P. Sculley, D. Yuan, R. Krishna and H. C. Zhou, J. Phys. Chem. C, 2013, 117, 4057-4061.

16 J. Caro and M. Noack, Microporous Mesoporous Mater., 2008, 115, 215-233.

17 R. Krishna and J. M. van Baten, J. Membr. Sci., 2013, 430, 113-128.

18 F. J. Keil, R. Krishna and M. O. Coppens, Rev. Chem. Eng., 2000, 16, 71-197.

19 D. Dubbeldam and R. Q. Snurr, Mol. Simul., 2007, 33, 15-30.

20 R. Krishna, J. Phys. Chem. C, 2009, 113, 19756-19781.

21 R. Krishna, Chem. Soc. Rev., 2012, 41, 3099-3118.

22 C. Chmelik and J. Kärger, Chem. Soc. Rev., 2010, 39, 4864-4884.

23 H. Jobic and D. N. Theodorou, Microporous Mesoporous Mater., 2006, 102, 21-50.

24 B. Smit and T. L. M. Maesen, Chem. Rev., 2008, 108, 4125-4184.

25 R. M. Barrer and J. W. Sutherland, Proc. R. Soc. London, Ser. A, 1956, 237, 439-463.

26 T. C. Golden and S. Sircar, J. Colloid Interface Sci., 1994, 162, 182-188.

27 S. Li, J. L. Falconer, R. D. Noble and R. Krishna, Ind. Eng. Chem. Res., 2007, 46, 3904-3911.

28 S. Li, J. L. Falconer, R. D. Noble and R. Krishna, J. Phys. Chem. C, 2007, 111, 5075-5082.

29 R. Krishna and J. M. van Baten, Chem. Eng. Sci., 2012, 69, 684-688.

30 R. Krishna and J. M. van Baten, J. Phys. Chem. C, 2010, 114, 11557-11563.

31 R. Krishna and J. M. van Baten, AIChE J., 2010, 56, 3288-3289.

32 R. Krishna and J. M. van Baten, Chem. Eng. Sci., 2009, 64, 3159-3178.

33 R. Krishna and J. M. van Baten, Ind. Eng. Chem. Res., 2011, 50, 7083-7087.

34 R. Krishna and J. M. van Baten, Chem. Eng. Sci., 2009, 64, 870-882. 
35 R. Krishna and J. M. van Baten, Chem. Eng. Sci., 2008, 63, 3120-3140.

36 G. K. Papadopoulos, H. Jobic and D. N. Theodorou, J. Phys. Chem. B, 2004, 108, 12748-12756.

37 D. A. Reed and G. Ehrlich, Surf. Sci., 1981, 102, 588-609.

38 R. Krishna and J. M. van Baten, Microporous Mesoporous Mater., 2008, 109, 91-108.

39 R. Krishna, D. Paschek and R. Baur, Microporous Mesoporous Mater., 2004, 76, 233-246.

40 R. Krishna and J. M. van Baten, Chem. Eng. Technol., 2005, 28, 160-167.

41 H. Wu, J. M. Simmons, G. Srinivas, W. Zhou and T. Yildrim, J. Phys. Chem. Lett., 2010, 1, 1946-1951.

42 R. Krishna and J. M. van Baten, J. Membr. Sci., 2011, 377, 249-260.

43 E. Beerdsen, D. Dubbeldam and B. Smit, J. Phys. Chem. B, 2006, 110, 22754-22772.

44 E. Beerdsen, D. Dubbeldam and B. Smit, Phys. Rev. Lett., 2006, 96, 044501.

45 J. Caro, M. Bülow, W. Schirmer, J. Kärger, W. Heink and H. Pfeifer, J. Chem. Soc., Faraday Trans., 1985, 81, 2541-2550.

46 D. R. Garg and D. M. Ruthven, Chem. Eng. Sci., 1972, 27, 417-423.

47 R. Krishna and R. Baur, Sep. Purif. Technol., 2003, 33, 213-254.

48 R. Krishna and J. M. van Baten, Microporous Mesoporous Mater., 2011, 137, 83-91.

49 R. Krishna and J. M. van Baten, J. Phys. Chem. C, 2010, 114, 18017-18021.

50 D. M. Ruthven and S. C. Reyes, Microporous Mesoporous Mater., 2007, 104, 59-66.

51 R. Krishna and J. M. van Baten, J. Membr. Sci., 2010, 360, 323-333.

52 E. Haldoupis, T. Watanabe, S. Nair and D. S. Sholl, ChemPhysChem, 2012, 13, 3449-3452.

53 D. Peralta, G. Chaplais, A. Simon-Masseron, K. Barthelet and G. D. Pirngruber, Ind. Eng. Chem. Res., 2012, 51, 4692-4702.

54 C. Zhang, R. P. Lively, K. Zhang, J. R. Johnson, O. Karvan and W. J. Koros, J. Phys. Chem. Lett., 2012, 3, 2130-2134.

55 K. Li, D. H. Olson, J. Seidel, T. J. Emge, H. Gong, H. Zeng and J. Li, J. Am. Chem. Soc., 2009, 131, 10368-10369.

56 R. C. Runnebaum and E. J. Maginn, J. Phys. Chem. B, 1997, 101, 6394-6408.

57 K. Yoo, R. Tsekov and P. G. Smirniotis, J. Phys. Chem. B, 2003, 107, 13593-13596.

58 R. L. Gorring, J. Catal., 1973, 31, 13-26.

59 D. Dubbeldam, S. Calero, T. L. M. Maesen and B. Smit, Angew. Chem., Int. Ed., 2003, 42, 3624-3626.

60 D. Dubbeldam, S. Calero, T. L. M. Maesen and B. Smit, Phys. Rev. Lett., 2003, 90(24), 245901.

61 D. Dubbeldam and B. Smit, J. Phys. Chem. B, 2003, 107, 12138-12152.

62 H. Jobic, A. Methivier, G. Ehlers, B. Farago and W. Haeussler, Angew. Chem., Int. Ed., 2004, 43, 364-366.
63 R. Krishna and J. M. van Baten, Mol. Simul., 2009, 35, 1098-1104.

64 R. Krishna and J. M. van Baten, J. Phys. Chem. C, 2012, 116, 23556-23568.

65 X. Kong, E. Scott, W. Ding, J. A. Mason, J. R. Long and J. A. Reimer, J. Am. Chem. Soc., 2012, 134, 14341-14344.

66 Z. Bao, L. Yu, Q. Ren, X. Lu and S. Deng, J. Colloid Interface Sci., 2011, 353, 549-556.

67 D. J. Lee, Q. Li, H. Kim and K. Lee, Microporous Mesoporous Mater., 2012, 163, 169-177.

68 E. Beerdsen, D. Dubbeldam and B. Smit, Phys. Rev. Lett., 2005, 95, 164505.

69 A. Gupta, L. A. Clark and R. Q. Snurr, Langmuir, 2000, 16, 3910-3919.

70 C. Chmelik, L. Heinke, J. Kärger, D. B. Shah, W. Schmidt, J. M. van Baten and R. Krishna, Chem. Phys. Lett., 2008, 459, 141-145.

71 T. J. H. Vlugt, R. Krishna and B. Smit, J. Phys. Chem. B, 1999, 103, 1102-1118.

72 R. Krishna and J. M. van Baten, Chem. Phys. Lett., 2005, 407, 159-165.

73 M. Fernandez, J. Kärger, D. Freude, A. Pampel, J. M. van Baten and R. Krishna, Microporous Mesoporous Mater., 2007, 105, 124-131.

74 C. Förste, A. Germanus, J. Kärger, H. Pfeifer, J. Caro, W. Pilz and A. Zikánóvá, J. Chem. Soc., Faraday Trans. 1, 1987, 83, 2301-2309.

75 A. O. Koriabkina, A. M. de Jong, D. Schuring, J. van Grondelle and R. A. van Santen, J. Phys. Chem. B, 2002, 106, 9559-9566.

76 R. Krishna and J. M. van Baten, Langmuir, 2010, 26, 3981-3992.

77 R. Krishna and J. M. van Baten, Langmuir, 2010, 26, 8450-8463.

78 R. Krishna and J. M. van Baten, Microporous Mesoporous Mater., 2011, 142, 745-748.

79 J. Getzschmann, I. Senkovska, D. Wallacher, M. Tovar, D. Fairen-Jimenez, T. Düren, J. M. van Baten, R. Krishna and S. Kaskel, Microporous Mesoporous Mater., 2010, 136, 50-58.

80 C. Chmelik, J. Kärger, M. Wiebcke, J. Caro, J. M. van Baten and R. Krishna, Microporous Mesoporous Mater., 2009, 117, 22-32.

81 F. Salles, H. Jobic, A. Ghoufi, P. L. Llewellyn, C. Serre, S. Bourrelly, G. Férey and G. Maurin, Angew. Chem., Int. Ed., 2009, 48, 8335-8339.

82 F. Salles, H. Jobic, T. Devic, P. L. Llewellyn, C. Serre, G. Férey and G. Maurin, ACS Nano, 2010, 4, 143-152.

83 W. Heink, J. Kärger, S. Ernst and J. Weitkamp, Zeolites, 1994, 14, 320-325.

84 A. Germanus, J. Kärger and H. Pfeifer, Zeolites, 1985, 5, 91-95.

85 S. Brandani, D. M. Ruthven and J. Kärger, Zeolites, 1995, 15, 494-495.

86 C. Zhang and X. Yang, Fluid Phase Equilib., 2005, 231, 1-10. 87 J. Kärger and H. Pfeifer, Zeolites, 1987, 7, 90-107. 
88 C. Chmelik, H. Bux, J. Caro, L. Heinke, F. Hibbe, T. Titze and J. Kärger, Phys. Rev. Lett., 2010, 104, 085902.

89 M. Tsotsalas, P. Hejcik, K. Sumida, Z. Kalay, S. Furukawa and S. Kitagawa, J. Am. Chem. Soc., 2013, 135, 4608-4611.

90 H. W. Habgood, Can. J. Chem., 1958, 36, 1384-1397.

91 J. Kärger and M. Bülow, Chem. Eng. Sci., 1975, 30, 893-896.

92 R. Krishna, Chem. Eng. Sci., 1990, 45, 1779-1791.

93 O. Talu, M. S. Sun and D. B. Shah, AIChE J., 1998, 44, 681-694.
94 H. Ban, J. Gui, L. Duan, X. Zhang, L. Song and Z. Sun, Fluid Phase Equilib., 2005, 232, 149-158.

95 H. Jobic, C. Laloué, C. Laroche, J. M. van Baten and R. Krishna, J. Phys. Chem. B, 2006, 110, 2195-2201.

96 K. S. Walton, A. R. Millward, D. Dubbeldam, H. Frost, J. J. Low, O. M. Yaghi and R. Q. Snurr, J. Am. Chem. Soc., 2008, 130, 406-407.

97 R. Krishna and J. M. van Baten, Langmuir, 2010, 26, 10854-10867. 